\title{
Markov extensions and conditionally invariant measures for certain logistic maps with small holes
}

\author{
Mark Demers \\ Fairfield University, mdemers@fairfield.edu
}

Follow this and additional works at: https://digitalcommons.fairfield.edu/mathandcomputersciencefacultypubs

Copyright 2005 Cambridge University Press

\section{Peer Reviewed}

\section{Repository Citation}

Demers, Mark, "Markov extensions and conditionally invariant measures for certain logistic maps with small holes" (2005). Mathematics Faculty Publications. 36.

https://digitalcommons.fairfield.edu/mathandcomputerscience-facultypubs/36

\section{Published Citation}

Mark Demers, "Markov extensions and conditionally invariant measures for certain logistic maps with small holes," Ergodic Theory and Dynamical Systems 25:4 (2005), 1139-1171.

This Article is brought to you for free and open access by the Mathematics Department at DigitalCommons@Fairfield. It has been accepted for inclusion in Mathematics Faculty Publications by an authorized administrator of DigitalCommons@Fairfield. For more information, please contact digitalcommons@fairfield.edu. 


\title{
Markov extensions and conditionally invariant measures for certain logistic maps with small holes
}

\author{
MARK F. DEMERS \\ Department of Mathematics, Georgia Institute of Technology, Atlanta, GA 30332, USA \\ (e-mail:demers@math.gatech.edu)
}

(Received 6 June 2004 and accepted in revised form 19 October 2004)

\begin{abstract}
We study the family of quadratic maps $f_{a}(x)=1-a x^{2}$ on the interval $[-1,1]$ with $0 \leq a \leq 2$. When small holes are introduced into the system, we prove the existence of an absolutely continuous conditionally invariant measure using the method of Markov extensions. The measure has a density which is bounded away from zero and is analogous to the density for the corresponding closed system. These results establish the exponential escape rate of Lebesgue measure from the system, despite the contraction in a neighborhood of the critical point of the map. We also prove convergence of the conditionally invariant measure to the SRB measure for $f_{a}$ as the size of the hole goes to zero.
\end{abstract}

\section{Introduction}

Consider a particle on a billiard table with convex boundaries so that the dynamics of the particle are hyperbolic, i.e. the trajectories are unstable with respect to initial conditions. Suppose a small hole is made in the table. What are the statistical properties of the trajectories in this system? If $p_{n}$ is the probability that a trajectory remains on the table until time $n$, what is the decay rate of $p_{n}$ ? More generally, we can place a particle randomly on the table according to an initial distribution $\mu_{0}$. If $\mu_{n}$ represents its normalized distribution at time $n$ (assuming the particle has not escaped by time $n$ ), does $\mu_{n}$ converge to some $\mu$ independent of $\mu_{0}$ ? Such a measure $\mu$ is a conditionally invariant measure for the open billiard system.

Considering the billiard table with a small hole as a perturbation of the billiard table with no holes, we can pose a related question in terms of the stability of the closed system: does the conditionally invariant measure of the open system converge to the invariant measure of the closed system as the size of the hole tends to zero?

The billiard table with a hole as a model for an open chaotic dynamical system was proposed by Pianigiani and Yorke [PY]. Although these questions remain open, dynamical systems with holes have been studied in some detail. Mathematical results 
so far have focused on open systems which are uniformly hyperbolic. Pianigiani and Yorke [PY] and later Collet et al [CMS1, CMS2] studied expanding maps which admit a finite Markov partition after the introduction of holes. These results were generalized to smooth Smale horseshoes [C1, C2] and a class of scattering billiards with a non-eclipsing condition [LM, R]. Recently, Chernov and Markarian [CM2, CM1] studied Anosov diffeomorphisms with holes which were elements of a finite Markov partition. In [CMT1] and [CMT2], the Markov restriction on the holes was relaxed, but the results still used strongly the Markov partitions associated with Anosov diffeomorphisms.

In low-dimensional settings, efforts to drop the Markov requirements on both the map and the holes have had some success for expanding maps of the interval. A spectral analysis of the transfer operator was performed in $[\mathbf{B K}]$ and the stability of the spectrum was established in $[\mathbf{K L}]$ for perturbations of expanding maps including small holes. More constructive techniques using bounded variation and contraction mapping arguments have been used in $[\mathbf{B C h}]$ and $[\mathbf{L i M}]$ to prove the existence and properties of conditionally invariant measures. Markov extensions were used in [D] to drop some of the earlier technical requirements and limit only the size of the holes.

This brief survey highlights the classes of systems with holes which have been studied to date: expanding maps in one dimension; and in higher dimensions, systems which admit finite Markov partitions. These systems are all uniformly hyperbolic.

In this paper, we seek to understand the escape dynamics of a class of open systems which are not uniformly hyperbolic, but which do exhibit exponential recurrence times. To do this, we construct Markov extensions for certain parameter values of the logistic family after the introduction of holes. We then use the results obtained in [D] for abstract tower maps with holes to determine the existence and properties of a conditionally invariant measure.

In this use of Markov extensions, we follow the approach of Young in [Y2], in which Markov extensions were used to study a variety of closed systems including Axiom A diffeomorphisms, piecewise hyperbolic maps, Hénon maps, logistic maps, and a class of scattering billiards (see also [Y3, BY]). Chernov [Ch1, Ch2, Ch3] has also used this technique to study the statistical properties of other chaotic systems. By extending the use of Markov extensions to open systems, we hope to be able to study more general classes of systems with holes and in particular those which satisfy neither uniform hyperbolicity nor Markov requirements.

The outline of the paper is as follows. In $\S 2$ we introduce the class of logistic maps which we shall study in this paper and state our main results. Section 3 reviews the setting and main results for tower maps in [D] which we will use. In $\S 4$ we construct Markov extensions for our class of logistic maps with holes and in $\S 5$, we use the results of $\S 3$ to determine the existence and properties of a conditionally invariant measure.

1.1. Conditionally invariant measures. The problem of the billiard table with a hole can be reformulated for maps of the interval as follows.

Let $\hat{T}$ be a map of an interval $\hat{I}$ to itself. We take the hole $H$ to be a finite union of open intervals and keep track of the iterates of a point until it reaches the hole. Once a point enters $H$, it is not allowed to return. 
Let $I=\hat{I} \backslash H$ and let $T=\hat{T} \mid\left(I \cap \hat{T}^{-1} I\right)$. A probability measure $\mu$ on $\hat{I}$ is said to be conditionally invariant if

$$
\frac{\mu\left(T^{-1} A\right)}{\mu\left(T^{-1} I\right)}=\mu(A)
$$

for every Borel subset $A$ of $\hat{I}$. The measure $\mu$ is called an absolutely continuous conditionally invariant measure (abbreviated a.c.c.i.m.) if it is absolutely continuous with respect to Lebesgue measure.

The quantity $\lambda=\mu\left(T^{-1} I\right)$ is called the eigenvalue of the measure and $-\log \lambda$ represents the exponential rate at which mass escapes from the system. From the point of view of physical observables, we are interested in conditionally invariant measures whose escape rate indicates the rate at which (normalized) Lebesgue measure escapes from the system. For this reason, in this paper we will restrict our attention to the existence and properties of absolutely continuous conditionally invariant measures.

\section{Setting and statement of results}

We begin by defining the class of logistic maps that we shall study in this paper.

2.1. A class of logistic maps. One class of logistic maps which has been studied in some detail are those satisfying the Misiurewicz condition: namely, that there are no attracting or semi-attracting periodic orbits. In this paper, we study parameter values of $a$ for which $f_{a}$ satisfies a slightly more generalized set of conditions. This approach follows that of Wang and Young in [WY2]. We define the class of maps $\mathcal{M}$ as follows.

Definition 2.1. The logistic map $f=f_{a}$ is in $\mathcal{M}$ if there exists a $\delta_{0}>0$ such that the following conditions hold.

(a) The critical orbit is bounded away from 0: $\operatorname{dist}\left(f^{n}(0), 0\right)>2 \delta_{0}$ for all $n>0$.

(b) Dynamics outside of $\left(-\delta_{0}, \delta_{0}\right)$ : there exist $\lambda_{0}>0, M_{0} \in \mathbb{Z}^{+}$and $0<c_{0} \leq 1$ such that:

(i) for all $n \geq M_{0}$, if $x, f(x), \ldots, f^{n-1}(x) \notin\left(-\delta_{0}, \delta_{0}\right)$, then $\left|\left(f^{n}\right)^{\prime}(x)\right| \geq e^{\lambda_{0} n}$;

(ii) for any $n$, if $x, f(x), \ldots, f^{n-1}(x) \notin\left(-\delta_{0}, \delta_{0}\right)$ and $f^{n}(x) \in\left(-\delta_{0}, \delta_{0}\right)$, then $\left|\left(f^{n}\right)^{\prime}(x)\right| \geq c_{0} e^{\lambda_{0} n}$.

(c) Recovery time for $x \in\left(-\delta_{0}, \delta_{0}\right)$ : for all $x \in\left(-\delta_{0}, \delta_{0}\right)$, there exists $s_{0}(x) \sim \log 1 /|x|$ such that $f^{j}(x) \notin\left(-\delta_{0}, \delta_{0}\right)$ for all $j<s_{0}$ and $\left|\left(f^{s_{0}}\right)^{\prime}(x)\right| \geq c_{0}^{-1} e^{\frac{1}{3} \lambda_{0} s_{0}}$.

Lemma 2.5 of [WY1] implies that maps satisfying the Misiurewicz condition belong to $\mathcal{M}$. In viewing this class of maps, we divide the phase space into two parts: $\left(-\delta_{0}, \delta_{0}\right)$ and its complement. Part (b) of the definition says that $f$ is essentially expanding outside of $\left(-\delta_{0}, \delta_{0}\right)$ while part (c) ensures that when orbits come close to the critical point, they subsequently spend enough time away from $\left(-\delta_{0}, \delta_{0}\right)$ for their derivatives to recover some exponential growth.

Although our method of proof will work for any map satisfying the above definition, for definiteness, we take $a$ near 2 in the proofs contained in $\S 4$. In this parameter range, we think of $\lambda_{0}$ as $\log 1.9$. 
2.2. Introduction of the hole. A hole $H$ in $[-1,1]$ is a finite union of open intervals $H_{j}$, $j=1, \ldots L$. We wish to study the dynamics of $f_{a} \in \mathcal{M}$ on $[-1,1] \backslash H$ and in particular to establish an exponential rate of escape from $[-1,1]$. To this end, we define $\hat{I}=[-1,1]$, $I=\hat{I} \backslash H$. We fix $a$, let $\hat{T}=f_{a}$ and set $I^{n}=\bigcap_{i=0}^{n} \hat{T}^{-i} I$. Let $T=\hat{T} \mid I^{1}$.

Our first assumption on the hole involves its location in $[-1,1]$.

Condition A1. The critical orbit is bounded away from $H$.

We define $r$ to be the smaller of this distance and $\delta_{0}$.

Our second condition on $H$ is that the positions of its components are generic with respect to one another.

Condition A2. For a fixed $m_{0} \in \mathbb{Z}^{+}$, there exists $\varepsilon_{0}>0$ such that for any interval $\omega \subset I$, if $\left|\hat{T}^{i} \omega\right|<\varepsilon_{0}$ for $0 \leq i \leq m_{0}$, then there is at most one $i$ and one $j$ such that $\hat{T}^{i} \omega \cap H_{j} \neq \emptyset$.

We are free to choose $m_{0}$ and generally $m_{0}$ will depend on $\lambda_{0}$. For $a$ near 2 , we mentioned earlier that $\lambda_{0}=\log 1.9$ and in this case $m_{0}=10$ is large enough.

Practically, Condition A2 may be difficult to check. However, if we let $N_{\varepsilon_{0}}(A)$ be the deleted $\varepsilon_{0}$-neighborhood of a set $A$, then Condition A 2 is implied by the simpler condition $N_{\varepsilon_{0}}\left(\hat{T}^{i} H\right) \cap N_{\varepsilon_{0}}\left(\hat{T}^{j} H\right)=\emptyset$ for every pair $i \neq j, 0 \leq i, j \leq m_{0}$.

The third assumption is on the size of the hole. We use $m$ interchangeably as Lebesgue measure on both the tower and on the interval $[-1,1]$. Let $\theta$ be the exponential rate of return given by Proposition $4.2, \varepsilon$ be the length scale of the reference intervals $\Lambda^{(i)}$ and $D$ be the constant in Lemma 4.4. Our assumption on the measure of the hole is the following.

Condition A3.

$$
m(H)<\frac{(1-\sqrt{\theta})^{3}}{3} \cdot \frac{\varepsilon^{2}}{D \theta} .
$$

As we will see in $\S 4, \varepsilon \sim \delta^{2}$ and $D \sim 1 / \delta$ so the restriction on the size of the hole is $\sim \delta^{5}$ where $\delta<\delta_{0}$ defines a neighborhood of the critical point which we shall use to keep track of intervals that pass near the critical point and subsequently need time for their derivatives to recover. Condition A3 in turn implies the following upper bound

$$
m(H) \leq \frac{\varepsilon}{2} \frac{e^{\frac{1}{3} \lambda_{0} m_{0}}-2}{e^{\frac{1}{3} \lambda_{0}\left(m_{0}-1\right)}} c_{0}^{\prime} \delta
$$

which we shall use to ensure that a part of every piece of length $\varepsilon$ is returned to the base of the tower. Condition A3 itself is used to ensure that the tower we construct satisfies Condition $\mathrm{H} 2$ of $\S 3$ on the measure of the holes in the tower. Equation (1) is implied by Condition A3 for small $\delta$ since it only requires that $m(H) \sim \delta^{3}$.

These three conditions will allow us to construct a Markov extension for $T$ with an exponential rate of return. In order to prove that the conditionally invariant measure obtained in $\$ 5.3$ is bounded away from zero, we need a transitivity condition. We shall use the following fact about maps in the class $\mathcal{M}$ :

$\exists n_{0}=n_{0}\left(\varepsilon_{0}\right)$ such that for every interval $J \subseteq \hat{I}$ with $|J| \geq \varepsilon_{0} / 2, \hat{T}^{n_{0}} J \supseteq[1-a, 1]$.

For each component of the hole $H_{j}, \hat{T}^{-1}\left(\hat{T} H_{j}\right)$ is comprised of two intervals: $H_{j}$ and its symmetric counterpart which we shall call $G_{j}$. The transitivity condition we need on the holes is stated in terms of the integer $n_{0}$ and the collection of intervals $H_{j}$ and $G_{j}$. 
Condition A4.

(a) $\hat{T}^{i} H_{j} \cap G_{k}=\emptyset$ for all $j, k \in[1, \ldots, L], 0 \leq i \leq n_{0}$.

(b) $\hat{T}^{i} H_{j} \cap H_{k}=\emptyset$ for all $j, k \in[1, \ldots, L], 1 \leq i \leq n_{0}$.

Taking $i=0$, we see that Condition A4(a) implies $\left\{T^{-1}(x)\right\} \neq \emptyset$ for every $x \in$ $[1-a, 1]$, i.e. the hole is not allowed to eliminate both preimages of any point. Given the small size of the holes, Condition A4 can be interpreted as requiring that the holes be in generic position with respect to one another.

\subsection{Statement of results.}

THEOREM 2.2. Given a logistic map $\hat{T} \in \mathcal{M}$ and a hole $H$ in $[-1,1]$ satisfying Conditions A1-A3, the open system $(T, I)$ has a Markov extension $(F, \Delta)$ with an exponential rate of return.

Projecting the a.c.c.i.m. for $(F, \Delta)$ onto $I$ yields the second theorem.

THEOREM 2.3. Under the conditions of Theorem 2.2, $T$ admits an absolutely continuous conditionally invariant measure on I. The density $\psi$ can be written as

$$
\psi=\rho_{1}+\rho_{2}
$$

where $\rho_{1}$ is of bounded variation and

$$
\rho_{2}(x) \leq \text { const. } \sum_{k=1}^{\infty} \frac{(1.9)^{-k / 3}}{\sqrt{x-\hat{T}^{k}(0)}} .
$$

If in addition Condition $A 4$ is satisfied, then $\psi$ is bounded away from zero on $[1-a, 1] \backslash H$.

We obtain convergence of these measures as the size of the hole goes to zero.

THEOREM 2.4. Let $\hat{T}$ and $H$ satisfy Conditions Al-A4. Define $H_{t}=H$ and let $\left\{H_{s}\right\}$ for $s \in[0, t]$ be a sequence of holes with the following properties:

(1) $m H_{s} \leq s, H_{s} \subset H_{t}$ and each component of $H_{t}$ contains at most one component of $H_{s}$;

(2) either $0, \delta,-\delta \notin H_{t}$ or $0, \delta,-\delta \in H_{s}$ for all $s \in[0, t]$.

Let $\mu_{s}$ be the a.c.c.i.m. corresponding to $H_{s}$ obtained from Theorem 2.3. As s goes to zero, the sequence $\mu_{s}$ converges weakly to the unique absolutely continuous invariant measure for $\hat{T}$ with no holes.

The conditions on the sequence of holes ensure that the intervals of monotonicity of the map $T$ do not decrease in length. This allows us to choose the same constants in our construction for each $s$ and so gain uniform estimates.

2.4. Some properties of maps in the class $\mathcal{M}$. Before beginning our construction of the Markov extension of a logistic map $\hat{T}$, we review some properties of maps in the class $\mathcal{M}$ defined in $\S 2.1$.

The following lemma is proved in [WY2]. We present the proof here for completeness. 
LEMMA 2.5. For $\hat{T} \in \mathcal{M}$, there exists $c_{0}^{\prime}>0$ such that the following hold for all $\delta<\delta_{0}$ :

(a) if $x, \hat{T}(x), \ldots, \hat{T}^{n-1}(x) \notin(-\delta, \delta)$, then $\left|\left(\hat{T}^{n}\right)^{\prime}(x)\right| \geq c_{0}^{\prime} \delta e^{\frac{1}{3} \lambda_{0} n}$;

(b) if $x, \hat{T}(x), \ldots, \hat{T}^{n-1}(x) \notin(-\delta, \delta)$ and $\hat{T}^{n}(x) \in\left(-\delta_{0}, \delta_{0}\right)$, then $\left|\left(\hat{T}^{n}\right)^{\prime}(x)\right| \geq$ $c_{0} e^{\frac{1}{3} \lambda_{0} n}$.

Proof. Let $x$ satisfy $\hat{T}^{i}(x) \notin(-\delta, \delta)$ for $i=1, \ldots, n-1$. Suppose $\hat{T}^{i}(x)$ enters $\left(-\delta_{0}, \delta_{0}\right)$ at times $t_{1}, \ldots, t_{s}$ before time $n$. Let $t_{0}=0$ and $t_{s+1}=n$ We set $k_{j}=t_{j+1}-t_{j}$ and estimate the derivative on each time interval $\left[t_{j}, t_{j+1}\right]$. There are four cases to consider.

Case 1. $\hat{T}^{t_{j}}(x), \hat{T}^{t_{j+1}}(x) \in\left(-\delta_{0}, \delta_{0}\right)$. Then $\left|\left(\hat{T}^{k_{j}}\right)^{\prime}\left(\hat{T}^{t_{j}} x\right)\right| \geq e^{\frac{1}{3} \lambda_{0} k_{j}}$ using Definition 2.1(b)(ii) and (c).

Case 2. $\hat{T}^{t_{j}}(x) \notin\left(-\delta_{0}, \delta_{0}\right), \hat{T}^{t_{j+1}}(x) \in\left(-\delta_{0}, \delta_{0}\right)$. Then $\left|\left(\hat{T}^{k_{j}}\right)^{\prime}\left(\hat{T}^{t_{j}} x\right)\right| \geq c_{0} e^{\lambda_{0} k_{j}}$ using Definition 2.1(b)(ii).

Case 3. $\hat{T}^{t_{j}}(x), \hat{T}^{t_{j+1}}(x) \notin\left(-\delta_{0}, \delta_{0}\right)$. If $k_{j} \geq M_{0}$, then $\left|\left(\hat{T}^{k_{j}}\right)^{\prime}\left(\hat{T}^{t_{j}} x\right)\right| \geq e^{\lambda_{0} k_{j}}$ by Definition 2.1(b)(i). Otherwise, $\left|\left(\hat{T}^{k_{j}}\right)^{\prime}\left(\hat{T}^{t_{j}} x\right)\right| \geq c_{0}^{\prime \prime} e^{\frac{1}{3} \lambda_{0} k_{j}}$ with $c_{0}^{\prime \prime}=\delta_{0}^{M_{0}-1} e^{-\frac{1}{3} \lambda_{0}\left(M_{0}-1\right)}$.

Case 4. $\hat{T}^{t_{j}}(x) \in\left(-\delta_{0}, \delta_{0}\right), \hat{T}^{t_{j+1}}(x) \notin\left(-\delta_{0}, \delta_{0}\right)$. This is the same as Case 3 with an additional factor $\geq \delta$.

Stringing these cases together, we obtain (b) using cases 1 and 2 and (a) with $c_{0}^{\prime}:=c_{0} \cdot c_{0}^{\prime \prime}$.

It may seem at first glance that the introduction of a new $\delta<\delta_{0}$ is redundant since there are analogous properties associated with each. The key difference, however, is that $\delta_{0}$ and the constants associated with it depend only on the map $\hat{T}$, whereas we are free to choose $\delta$. We shall choose $\delta$ depending on several factors involved in the construction of the tower as well as the placement of the hole.

We now explore a second property of maps in the class $\mathcal{M}$. This property concerns a period of recovery for $\left(\hat{T}^{j}\right)^{\prime}(x)$ for orbits which pass through a $\delta$-neighborhood of the critical point. Let $\delta=e^{-k_{0}}$ and define a partition of $(-\delta, \delta)$ into intervals $I_{k}=$ $\left(e^{-(k+1)}, e^{-k}\right), k \geq k_{0}$, and $I_{k}=-I_{-k}$ for $k \leq-k_{0}$. $k_{0}$ will be chosen large enough so that the series in the proof of Proposition 4.1 converge.

For $x \in I_{k}$, define $\tilde{p}(x)=\max \left\{n \in \mathbb{Z}:\left|\hat{T}^{j} x-\hat{T}^{j} 0\right|<1 / j^{2}\right.$, for all $\left.j<n\right\}$. Let

$$
p(x)=\inf _{y \in I_{k}} \tilde{p}(y) .
$$

The number $p(x)$ is called the bound period of $x$ by Benedicks and Carleson in [BC]. We call an interval $\omega \subset I_{k}$ bound from time 1 until time $p-1$ and free from time $p$ until $\omega$ enters $(-\delta, \delta)$ again. Then another bound period begins. Since $p$ is constant on each $I_{k}$, we sometimes refer to $p$ as $p(k)$. For $a$ near 2, the following properties of $p$ are proved in [BC] and outlined succinctly in [Y1].

Properties P1. The function $p:(-\delta, \delta) \rightarrow \mathbb{Z}^{+}$is constant on each $I_{k}$ and increasing with $|k|$. In addition, for $x \in I_{k}$ :

(a) $\quad \frac{1}{2}|k| \leq p(x) \leq 4|k|$

(b) $\left|\left(\hat{T}^{j}\right)^{\prime}(x)\right| \approx$ const. $\left|\left(\hat{T}^{j}\right)^{\prime}(\hat{T} 0)\right| \geq$ const. $(1.9)^{j}$, for all $j<p(x)$;

(c) $\left|\left(\hat{T}^{p}\right)^{\prime}(x)\right| \geq e^{p / 5}$.

The central distortion estimate which yields Properties P1 is given at the beginning of $\S 4.5$. 
We choose $\delta$ small enough so that $I_{k_{0}}$ is free by the time it leaves an $(r / 2)$-neighborhood of the critical orbit. This in turn implies that any interval $\omega \subset I_{k}$ must be free at time $n$ if $\hat{T}^{n} \omega$ intersects $H$. But we may conclude more than this. In fact, Condition A1 together with Lemma 4.5 ensures that each $I_{k}$ must grow to a fixed size before intersecting the hole. We call this fixed length $\varepsilon^{\prime}$.

We define time $q(k)$ for $k \geq k_{0}$ by

$$
q(k)=\max \left\{n \in \mathbb{Z}:\left|\hat{T}^{j}\left(0, e^{-k}\right)\right| \leq r / 2, \forall j \leq n\right\} .
$$

$q(k)$ is defined analogously for $k \leq-k_{0}$. Using Lemma 4.5 , we shall prove in $\$ 4.5$ that each $I_{k}$ must grow to length $\varepsilon^{\prime}$ by time $q(k)$. We also use $q(k)$ to define our construction of the stopping time $S$ and partition $\mathcal{Z}$ of Proposition 4.1. Note that $q(k) \geq p(k)$.

2.5. Markov extensions. We describe the main ideas of the construction of a Markov extension for maps of the interval following [Y3]. We carry out this construction in detail in $\S 4$.

Given a subinterval $\Lambda$ and a map $T$, we consider the forward images of $\Lambda$ under the action of $T$. When a connected component of $T^{n} \Lambda$ covers $\Lambda$, we declare $\omega$, the subinterval of $\Lambda$ satisfying $T^{n} \omega=\Lambda$, to have returned and stop iterating it. We continue to iterate the remaining components of $T^{n} \Lambda$ until they return to completely cover $\Lambda$. In this way, we generate a countable partition $\left\{\Lambda_{i}\right\}$ of subintervals of $\Lambda$ and a stopping time $R: \Lambda \rightarrow \mathbb{N}$, constant on elements of the partition and satisfying $T^{R}\left(\Lambda_{i}\right)=\Lambda$. Then $\left\{\Lambda_{i}\right\}$ is a countable Markov partition for the map $T^{R}$.

In this situation, we define a Markov extension of $T: \bigcup_{n \geq 0} T^{n} \Lambda \circlearrowleft$ as a dynamical system $F: \Delta \circlearrowleft$ for which there exists a projection $\pi: \Delta \rightarrow \bigcup_{n \geq 0} T^{n} \Lambda$ such that $\pi \circ F=T \circ \pi$.

We also call $F: \Delta \circlearrowleft$ the tower model or simply the tower associated with $T$. The reason for this is the following pictorial model for the Markov extension. Let $\Delta_{0}=\Lambda$ and define

$$
\Delta=\left\{(x, n) \in \Delta_{0} \times \mathbb{N}: R(x)>n\right\} .
$$

The tower map is given by

$$
F= \begin{cases}F(x, n)=(x, n+1) & \text { if } n+1<R(x), \\ F(x, n)=\left(T^{R}(x), 0\right) & \text { if } n+1=R(x) .\end{cases}
$$

The $l$ th level of the tower is $\left.\Delta\right|_{n=l}$ and the action of the tower map $F$ is to map a point up the levels of the tower until time $R$ at which time the point is returned to the base $\Delta_{0}$. Note that all of the returns to the base are Markov because of the nature of the returns of $\Lambda_{i}$ to $\Lambda$.

The flexibility of the Markov extension stems from the fact that the dynamical system in question need not be uniformly hyperbolic. What matters is the average behavior of the map $T$ between returns to $\Lambda$. This is what allows the method to be applied to Hénon maps and the logistic family. There are three basic steps which are required for this method to work: 
(1) given a dynamical system $T: M \circlearrowleft$, we construct a Markov extension $F: \Delta \circlearrowleft$;

(2) we prove results about $(F, \Delta)$ using its simpler properties: namely, controlled hyperbolicity and a countable Markov structure with a certain decay rate in the measure of the elements of the partition;

(3) we pass these results back to the original system $(T, M)$.

Step (1) is completed by the construction contained in $\S 4$. This is the most technical part of the paper. Step (2) is proved in [D] and those results are recalled in §3. Step (3) is completed in $\S 5$.

\section{Tower maps with holes}

The results of [D] for tower maps with holes apply in a more general setting than the present paper. Logistic maps are $C^{2}$ and the tower which we construct will have no holes in its base. Here we recall only those results relevant to our case. This simplifies the assumptions on the tower somewhat. We do, however, retain the definition of the function space $X$ in which the conditionally invariant density lies since we will use this to establish the properties of the conditionally invariant density for the logistic map in $\S 5$.

3.1. Tower with multiple bases. The towers studied in $[\mathbf{D}]$ are towers with multiple bases $\hat{\Delta}_{0}^{(i)}$, which are intervals of unit length whose interiors are pairwise disjoint. The base $\hat{\Delta}_{0}=\bigcup_{i=1}^{N} \hat{\Delta}_{0}^{(i)}$ is also an interval. We let $m$ denote one-dimensional Lebesgue measure on the tower and let $\mathcal{Z}$ be a countable partition of $\hat{\Delta}_{0}$ whose elements are subintervals of the $\hat{\Delta}_{0}^{(i)}$. Given a return time function $R: \hat{\Delta}_{0} \rightarrow \mathbb{Z}^{+}$which is constant on each element of $\mathcal{Z}$, a tower $(\hat{\Delta}, \hat{F}, m)$ is built over $\hat{\Delta}_{0}$ with

$$
\hat{\Delta}:=\left\{(z, n) \in \hat{\Delta}_{0} \times \mathbb{N}: n<R(z)\right\} .
$$

As before, we call the $l$ th level of the tower $\hat{\Delta}_{l}:=\left.\hat{\Delta}\right|_{n=l}$ and $\hat{\Delta}_{l}^{(i)}$ is the part of $\hat{\Delta}_{l}$ directly over $\hat{\Delta}_{0}^{(i)}$. We let $\hat{\Delta}^{(i)}=\bigcup_{l=0}^{\infty} \hat{\Delta}_{l}^{(i)}$.

The action of $\hat{F}: \hat{\Delta} \rightarrow \hat{\Delta}$ is $\hat{F}(z, l)=(z, l+1)$ if $l+1<R(z)$ and $\hat{F}^{R(z)}(\mathcal{Z}(z))=\hat{\Delta}_{0}^{(i)}$ for some $1 \leq i \leq N$, where $\left.\hat{F}^{R(z)}\right|_{\mathcal{Z}(z)}$ is continuous and one-to-one and $\mathcal{Z}(z)$ is the element of $\mathcal{Z}$ containing $z$.

The first assumption made on the tower is that the measure of the levels of the tower decays exponentially. This is crucial to the existence of an a.c.c.i.m. with good properties.

Condition H1. There exist $A>0$ and $0<\theta<1$ such that $m\left(\hat{\Delta}_{l}\right) \leq A \theta^{l}$ for $l \geq 0$.

We leave assumptions about the regularity of $\hat{F}$ until after we have introduced the holes.

3.2. Introduction of holes and regularity of $\hat{F}$. A hole $\tilde{H}$ in $\hat{\Delta}$ is a union of open intervals $\tilde{H}_{l, j}^{(i)}$ such that $\bigcup_{j} \tilde{H}_{l, j}^{(i)}=: \tilde{H}_{l}^{(i)} \subset \hat{\Delta}_{l}^{(i)}$ with finitely many $\tilde{H}_{l, j}^{(i)}$ per level $l$. We set $\tilde{H}_{l}=\bigcup_{i=1}^{N} \tilde{H}_{l}^{(i)}$. We require that each $\tilde{H}_{l, j}^{(i)}=\hat{F}^{l}(\omega)$, where $\omega$ is the union of elements of $\mathcal{Z}$, thus preserving the Markov structure of the returns to $\hat{\Delta}_{0}$. If $\hat{F}^{l}(\omega)=\tilde{H}_{l, j}^{(i)}$, then the intervals on all levels of the tower directly above $\tilde{H}_{l, j}^{(i)}$ are deleted since once $\hat{F}$ maps a point into $\tilde{H}$, it disappears forever. $\omega$ does not return to $\hat{\Delta}_{0}$. 
Let $\Delta=\hat{\Delta} \backslash \tilde{H}$ and $\Delta_{l}=\hat{\Delta}_{l} \backslash \tilde{H}$ with analogous definitions for $\Delta^{(i)}$ and $\Delta_{l}^{(i)}$. We assume the existence of a countable Markov partition $\left\{\Delta_{l, j}^{(i)}\right\}$ with $\bigcup_{j} \Delta_{l, j}^{(i)}=\Delta_{l}^{(i)}$ for each $i$ and $l$. Each $\Delta_{l, j}^{(i)}$ is an interval comprised of countably many elements of the form $\hat{F}^{l}(\omega), \omega \in \mathcal{Z}$, and $\left.\hat{F}\right|_{\Delta_{l, j}^{(i)}}$ is one-to-one.

In applications, $\left\{\Delta_{l, j}^{(i)}\right\}$ is dynamically defined during the construction of the tower and its elements are the maximal intervals which project onto the iterated pieces of the reference set $\Lambda$ at time $l$. For this reason, it is useful to keep track of the elements $\Delta_{l, j}^{(i)}$ rather than the elements of $\hat{F}^{l}(\mathcal{Z})$.

We denote by $\Delta_{l, j}^{(i) *}$ those $\Delta_{l, j}^{(i)}$ whose image returns to the base, i.e. such that $\hat{F}\left(\Delta_{l, j}^{(i)}\right)=$ $\bigcup_{k=k_{1}}^{k_{2}} \Delta_{0}^{(k)}$ for some $1 \leq k_{1} \leq k_{2} \leq N$, and set $\Delta^{*}=\bigcup \Delta_{l, j}^{(i) *}$.

Properties P2. Since $\hat{T}$ is $C^{2}$, the map $\hat{F}$ has the following properties with respect to the partition $\left\{\Delta_{l, j}^{(i)}\right\}$.

(a) $\hat{F}$ is $C^{2}$ on each $\Delta_{l, j}^{(i)}$.

(b) There exist $\gamma>1$ and $\beta>0$ such that on $\Delta_{l, j}^{(i) *},\left|\hat{F}^{\prime}\right| \geq \gamma e^{\beta l}$. Elsewhere, $\left|\hat{F}^{\prime}\right|=1$.

(c) Bounded Distortion. There exists $C>0$ such that for any $x, y \in \hat{\Delta}_{0}^{(i)}$ and $x^{\prime}, y^{\prime} \in \Delta_{l, j}^{(k) *}$ such that $\hat{F}\left(x^{\prime}\right)=x$ and $\hat{F}\left(y^{\prime}\right)=y$ we have

$$
\left|\frac{\hat{F}^{\prime}\left(x^{\prime}\right)}{\hat{F}^{\prime}\left(y^{\prime}\right)}-1\right| \leq C|x-y| .
$$

Controlling the distortion for logistic maps requires a countable partition in a neighborhood of the critical point. Such a partition has been introduced in $\$ 2.4$ and equation (2) is a consequence of the distortion lemmas of $\S 4.5$.

Let $F=\hat{F} \mid\left(\Delta \backslash \hat{F}^{-1} \tilde{H}\right)$. We say $F$ is transitive on components if for all pairs $i, j$, $1 \leq i, j \leq N$, there exists an $m$ such that $F^{m} \Delta_{0}^{(i)} \supseteq \Delta_{0}^{(j)}$. Note that if $N=1$, then transitivity on components is automatic as long as the hole allows at least one return to the base.

3.3. Definition of a convex functional. The Perron-Frobenius operator associated with $F$ acts on $L^{1}(\Delta)$ by

$$
\mathcal{P} f(x)=\sum_{y \in F^{-1} x} \frac{f(y)}{\left|F^{\prime}(y)\right|} .
$$

We define $\mathcal{P}_{1} f=\mathcal{P} f /|\mathcal{P} f|_{L^{1}}$ and seek a fixed point for the operator $\mathcal{P}_{1}$. A fixed point for $\mathcal{P}_{1}$ is a conditionally invariant density for $F$.

Choose $\xi>0$ small enough so that $e^{-\xi}>\max \left\{\theta, e^{-\beta}\right\}$. Given $f \in L^{1}(\Delta)$, let $f_{l, j}^{(i)}=\left.f\right|_{\Delta_{l, j}^{(i)}}$. Let $|f|_{\infty}$ denote the $L^{\infty}$ norm of $f$ and define

$$
\left\|f_{l, j}^{(i)}\right\|_{\infty}=\left|f_{l, j}^{(i)}\right|_{\infty} e^{-\xi l}, \quad\left\|f_{l, j}^{(i)}\right\|_{r}=\sup _{\substack{x \in \Delta_{l, j}^{(i)} \\ f(x) \neq 0}}\left|\frac{f^{\prime}(x)}{f(x)}\right| e^{-\xi l} .
$$


Then define

$$
\|f\|=\max \left\{\|f\|_{\infty},\|f\|_{r}\right\},
$$

where $\|f\|_{\infty}=\sup _{i, l, j}\left\|f_{l, j}^{(i)}\right\|_{\infty}$ and $\|f\|_{r}=\sup _{i, l, j}\left\|f_{l, j}^{(i)}\right\|_{r}$. Let $X=\{f: \Delta \rightarrow \mathbb{C}$ । $\|f\|<\infty$. Although $\|\cdot\|_{r}$ is not a norm, it does satisfy a convex-like inequality on a subset $X_{M}$ of $X$ defined by

$$
X_{M}=\left\{f \in X \mid\|f\| \leq M, f \geq 0, \int_{\Delta} f d m=1\right\} .
$$

It is proved in [D] that $X_{M}$ is a convex, compact subset of $L^{1}(\Delta)$. We take $M=b /\left(1-a_{0}\right)$, where $a_{0}$ and $b$ are defined below.

3.4. Condition on the holes and main result. We formulate a single condition involving the measure of the holes which guarantees the existence of an a.c.c.i.m. in X.

Let $a_{0}:=\max \left\{e^{-\xi}, 1 / \gamma\right\}$ and $b:=1+C$. The required condition on the holes is as follows:

\section{Condition H2.}

$$
\sum_{l \geq 1} e^{\xi(l-1)} m \tilde{H}_{l} \leq \frac{\left(1-a_{0}\right)^{2}}{b}
$$

The main result we wish to recall from [D] is the following theorem. We will apply this theorem in $\$ 5$ after constructing the Markov extension.

Theorem 3.1. Given a tower with holes $(\Delta, F, m)$ with Properties $P 2$ and under Conditions $H 1$ and H2, there exists a probability density $\varphi$ in $X_{M}$ such that $\mathcal{P}_{1} \varphi=\varphi$. If in addition $F$ is transitive on components, then $\varphi$ is the unique non-trivial conditionally invariant density in $X$ and $\varphi$ is bounded away from zero on $\Delta$.

Remark. Note that since $\varphi \in X$, its eigenvalue $\lambda$ must satisfy $\lambda \geq e^{-\xi}$. In fact, in [D] it is proven that $\lambda \geq 1-M \sum_{l \geq 1} e^{\xi(l-1)} m \tilde{H}_{l}$. This estimate stems from the lower bound on the renormalization constant for functions in the set $X_{M}$.

\section{Construction of the tower}

In this section we describe the construction of the Markov extension of $T: I^{1} \rightarrow I$. The construction entails finding the right length scale for the reference intervals $\Lambda^{(i)}$ which will constitute the base of the tower and showing that the object we construct has certain properties. These are summarized in Proposition 4.2 in $\$ 4.3$ and Proposition 4.3 in $\$ 4.4$.

The construction of the tower involves a series of constants which we define below. Some have been introduced already. The order of their selection is important and follows that of the list.

- $\quad$ The constants $\delta_{0}$ and $\lambda_{0}$ introduced in Definition 2.1. Throughout the proofs of $\S 4$, for definiteness we consider $\lambda_{0}$ as $\log 1.9$.

- The minimum distance $r$ between $H$ and the critical orbit introduced by Condition Al.

- $\quad m_{0}$ (depending on $\left.\lambda_{0}\right)$ and $\varepsilon_{0}$ introduced in Condition A2. If $\lambda_{0}$ is taken to be $\log 1.9$, then $m_{0}=10$ is large enough. 
- $\quad n_{0}$ (depending on $\epsilon_{0}$ ) is the least $i$ for which $\hat{T}^{i} J \supseteq[1-a, 1]$ for every interval $J$ of length at least $\varepsilon_{0} / 2 . \quad n_{0}$ is used in Condition A4 and later in $\$ 5.3$ to prove a transitivity property for the map with holes.

- $\delta=e^{-k_{0}}$, which defines a $\delta$-neighborhood of the critical point and induces the partition $\left\{I_{k}\right\}_{|k| \geq k_{0}}$ defined in $\$ 2.4$. $\delta$ is chosen small enough to make the series in the proof of Proposition 4.1 converge and also so that $I_{k_{0}}$ is free by the time it leaves an $(r / 2)$-neighborhood of the critical orbit.

- $\quad \varepsilon^{\prime}$, the fixed length to which every $I_{k}$ must grow by time $q(k)$ before intersecting the hole, proven after Lemma 4.5.

- $\quad \varepsilon$, the length of the reference intervals $\Lambda^{(i)}$ which constitute the base of the tower. $\varepsilon$ is chosen so that $4^{8} \varepsilon=\min \left\{\varepsilon^{\prime}, \varepsilon_{0}, 1 / 4 \tilde{C}\right\}$ where $\tilde{C}$ is the nonlinearity constant in the distortion estimate of Lemma 4.6. Since $\tilde{C} \sim 1 / \delta^{2}$, this requires $\varepsilon \sim \delta^{2}$. $\varepsilon$ is chosen to be small compared to $\varepsilon^{\prime}$ and $\varepsilon_{0}$ in order to control the rate at which pieces are generated during the construction of the tower. The requirement involving $\tilde{C}$ ensures a minimum expansion at the return time.

We begin by defining a partition $\mathcal{Q}$ and a type of interval $\Omega$ which we shall use in our construction.

Recall the partition of $(-\delta, \delta)$ introduced earlier: $\left\{I_{k}\right\}_{|k| \geq k_{0}}$. To this partition we join the partition of $[-1,1]$ into the finitely many maximal intervals of $I$ and $H$. We call this new partition $\mathcal{Q}$.

Let $\Omega$ be an interval such that $\varepsilon \leq|\Omega| \leq 3 \varepsilon$. We require that $\Omega \subset I$ and that either $\Omega \subset(-\delta, \delta) \backslash\{0\}$ or $\Omega \subset I \backslash(-\delta, \delta)$.

We cover $[1-a, 1] \backslash H$ with intervals $\Lambda^{(1)}, \ldots, \Lambda^{(N)}$, each of which is of the type $\Omega$ described above, except that we restrict $\varepsilon \leq\left|\Lambda^{(i)}\right| \leq 2 \varepsilon$. The intervals $\Lambda^{(i)}$ are the reference intervals which will serve as the base of the tower.

4.1. Introduction of an auxiliary stopping time. Let $\Omega$ be an interval of the form described above. The principal properties of the auxiliary stopping time and partition that we shall construct on $\Omega$ are listed in the following proposition.

PROpOSITION 4.1. There exist a countable partition $\mathcal{Z}$ of $\Omega$ and a stopping time $S$ satisfying:

(a) $S$ is constant on each element $\omega \in \mathcal{Z}$;

(b) $\quad T^{S} \omega$ is defined and $\left|T^{S} \omega\right| \geq 4^{8} \varepsilon$ or $T^{S-1} \omega$ is defined and $\hat{T}^{S} \omega \subset H$;

(c) There exits $\tilde{C} \sim 1 / \delta^{2}$ such that for $x, y \in \omega$,

$$
\left|\frac{\left(\hat{T}^{S}\right)^{\prime}(x)}{\left(\hat{T}^{S}\right)^{\prime}(y)}-1\right| \leq \tilde{C}\left|\hat{T}^{S}(x)-\hat{T}^{S}(y)\right| ;
$$

(d) $\left|\left(\hat{T}^{S}\right)^{\prime}(x)\right|>4^{6}$;

(e) $\quad m\{x \in \Omega: S(x)>n\} \leq C^{\prime} e^{-n / 21}$ for some $C^{\prime}$ independent of $\delta$.

The proof of this proposition in $\$ \S 4.1$ and 4.2 follows closely the approach of Benedicks and Young [BY] for Hénon maps without holes.

We construct $\mathcal{Z}$ and $S$ as follows. We take components of $\mathcal{Q} \mid \Omega$ and place them in the set $\Omega_{0}$. Given $\Omega_{n-1} \subset \Omega$, we proceed inductively. Let $\omega \in \Omega_{n-1}$. Let $t$ be the last time $\omega$ 
passed through $(-\delta, \delta)$ and let $k$ be such that $\hat{T}^{t} \omega \subset I_{k}$. If $\omega$ has not yet passed through $(-\delta, \delta)$ by time $n$, set $t=q(k)=0$.

If $n>t+q(k)$, we look at $\hat{T}^{n} \omega$ and do the following.

Case 1. $\hat{T}^{n} \omega$ does not intersect the hole. If $\left|\hat{T}^{n} \omega\right| \geq 4^{8} \varepsilon$, then enter $\omega$ as an element of $\mathcal{Z}$ and declare the stopping time $S(x) \equiv n$ on $\omega$. Otherwise partition $\omega$ according to $\hat{T}^{-n} \mathcal{Q} \mid \omega$ and put these pieces into $\Omega_{n}$. If $\hat{T}^{n} \omega$ lies partly outside and partly inside of $(-\delta, \delta)$ then we append the piece lying outside to the piece of $\hat{T}^{n} \omega$ lying in $I_{ \pm k_{0}}$ and do not introduce a cut there. Since $\varepsilon \sim \delta^{2}$, this added length is negligible from time $n$ to time $n+q\left(k_{0}\right)$.

Case 2. $\hat{T}^{n} \omega$ intersects the hole. Set $S(x) \equiv n$ on the components of $\omega \cap \hat{T}^{-n} H$ and enter them as elements of $\mathcal{Z}$. Take the remaining subintervals of $\omega$ and follow the procedure described in Case 1 for each. Note that there can be at most two subintervals $\omega_{n}$ of $\omega$ such that $\left|\hat{T}^{n} \omega_{n}\right|<4^{8} \varepsilon$ because of Condition A2 and our choosing $4^{8} \varepsilon \leq \varepsilon_{0}$.

If $n<t+q(k)$, then $\mathcal{Q} \mid \hat{T}^{n} \omega$ will have only one component. We put $\omega$ in $\Omega_{n}$ and continue to iterate it.

If $n=t+q(k)$, then if $\left|\hat{T}^{n} \omega\right|<4^{8} \varepsilon$, we put $\omega \in \Omega_{n}$. If $\left|\hat{T}^{n} \omega\right| \geq 4^{8} \varepsilon$, we do one of two things.

Case 1. $\omega \in \Omega_{t-1}$. Then $\omega$ was not created by a cut at time $t$. We declare $S(x) \equiv n$ on $\omega$ and enter $\omega$ as an element of $\mathcal{Z}$.

Case 2. $\omega \notin \Omega_{t-1}$. Then $\omega$ was created at time $t$ by a cut between $I_{k}$ and $I_{k+1}$. So there are two intervals $\omega$ and $\gamma$ such that $\omega \cup \gamma$ is one interval until time $t, \hat{T}^{t} \omega \subseteq I_{k}$ and $\hat{T}^{t} \gamma \subseteq I_{k+1}$; but $\hat{T}^{n} \omega$ and $\hat{T}^{n} \gamma$ are still adjacent. $\hat{T}^{n} \omega$ will overlap a large number of the $\Lambda^{(i)}$. On the side of $\omega$ adjacent to $\gamma$, we adjoin to $\gamma$ the part of $\omega$ which does not completely cover the last $\Lambda^{(i)}$ on that side under $\hat{T}^{n}$. Let us call this interval $\omega^{\prime}$. We declare $S(x) \equiv n$ on $\omega \backslash \omega^{\prime}$ and put the interval $\omega^{\prime} \cup \gamma$ into $\Omega_{n}$ and continue to iterate it. We do this to control the number of pieces generated by the process described later in $\S 4.3$. We will need this control in order to obtain the bounds on the conditionally invariant density in $§ 5.4$. (Note that if $\omega$ had been created by a cut between $I_{k}$ and $I_{k-1}$, the process of adjoining a left over piece on that side would already have occurred at time $t+q(k-1)$.)

It is clear that Proposition 4.1(a) and (b) will be satisfied by the construction described above. Item (c) is proven by the distortion bounds of Lemma 4.6 and item (d) will follow immediately from that. Item (e) is proved in $\S 4.2$.

We close this section by showing that every interval of length at least $\varepsilon$ will grow to length $4^{8} \varepsilon$ using the upper bound on the size of the hole given by equation (1). Suppose $\Omega$ is an interval of length at least $\varepsilon$ and suppose that $\Omega$ intersects the hole after its very first iterate. Then there will be at most two pieces of $\Omega$ whose image did not fall into the hole. Choose the longer of the two pieces and call it $\omega_{1}$. Using Lemma 2.5(a), we observe that

$$
\left|\omega_{1}\right| \geq \frac{1}{2}\left(\varepsilon-\frac{m H}{c_{0}^{\prime} \delta e^{\frac{1}{3} \lambda_{0}}}\right) .
$$

If $\omega_{1}$ does not grow to length $4^{8} \varepsilon$, it must wait at least another $m_{0}$ iterates before intersecting $H$ again. Say this happens at time $t_{1}$. Once again, there are at least two pieces of $\omega_{1}$ whose images do not intersect the hole under $\hat{T}^{t_{1}}$. Call the longer of these $\omega_{2}$ 
and note that

$$
\left|\omega_{2}\right| \geq \frac{1}{2}\left(\frac{1}{2}\left(\varepsilon-\frac{m H}{c_{0}^{\prime} \delta e^{\frac{1}{3} \lambda_{0}}}\right)-\frac{m H}{c_{0}^{\prime} \delta e^{\frac{1}{3} \lambda_{0}\left(m_{0}+1\right)}}\right) .
$$

Repeating this process $k$ times and always following the larger half, we see that

$$
\left|\omega_{k}\right| \geq \frac{\varepsilon}{2^{k}}-\sum_{i=0}^{k-1} \frac{m H}{c_{0}^{\prime} \delta e^{\frac{1}{3} \lambda_{0}\left(i m_{0}+1\right)} 2^{k-i}} \geq \frac{\varepsilon}{2^{k+1}},
$$

where we have used equation (1) in the last step. Following this process until time $n$, and noting that $n \geq m_{0} k$, we have

$$
\left|\hat{T}^{n} \omega_{k}\right| \geq c_{0}^{\prime} \delta e^{\frac{1}{3} \lambda_{0} n} \frac{\varepsilon}{2^{\left(n / m_{0}\right)+1}}
$$

which is exponentially increasing. This will continue until a part of $\Omega$ grows to length $4^{8} \varepsilon$. If along the way, $\hat{T}^{n} \omega_{k}$ lands in $(-\delta, \delta)$, then our estimates only improve since the piece cannot intersect the hole again until the partition element it lies in grows to size $\varepsilon^{\prime} \geq 4^{8} \varepsilon$.

4.2. Estimating the return time function $S$. In this section we prove that $m\{x \in \Omega$ : $S(x)>n\} \leq C^{\prime} e^{-n / 21}$, which is part (e) of Proposition 4.1 .

In order to estimate the tail of the return time function $S$, we will use information about the times when an interval passes through $(-\delta, \delta)$. Recall that for $\omega \in \Omega_{n-1}$ if $\hat{T}^{n} \omega$ intersects $(-\delta, \delta)$, then we introduce cuts in $\omega$ according to the partition $\hat{T}^{-n} \mathcal{Q} \mid \omega$ and the pieces are entered as elements of $\Omega_{n}$. We keep track of which interval $I_{k}$ each piece passes through at time $n$.

If an interval $\omega$ is a subset of $I_{r_{i}}$ at time $t_{i}, 1 \leq i \leq s$, then we say $\omega$ has itinerary $\left(r_{1}, \ldots, r_{s}\right)$. Let $p_{i}=p\left(I_{r_{i}}\right) .\left(p_{1}, \ldots, p_{s}\right)$ are the recovery times associated with the itinerary $\left(r_{1}, \ldots, r_{s}\right)$. Recall that if $\hat{T}^{n} \omega$ lies partly outside of $(-\delta, \delta)$ then we append the piece lying outside to the piece of $\hat{T}^{n} \omega$ lying in $I_{ \pm k_{0}}$ and do not introduce a cut there. This will not affect the recovery time $p\left( \pm k_{0}\right)$ of $I_{ \pm k_{0}}$.

Notice that by construction, pieces that are created by an interval landing on $(-\delta, \delta)$ at any given time will have different itineraries; however, if an interval is mapped across one of the holes $H_{j}$ and split into two pieces, then those two pieces may be mapped into $(-\delta, \delta)$ at different times and so generate separate pieces with the same itinerary. We wish to obtain an upper bound on the number of pieces with the same itinerary up to time $n$ that can be created from a single interval which is iterated according to the procedure described after the statement of Proposition 4.1.

Let $\omega \subset I_{r_{0}}$ and let $S_{n}$ be the set of elements of $\Omega_{n}$ which have the same itinerary $\left(r_{1}, \ldots, r_{s}\right)$ at time $n$. Now $I_{r_{0}}$ cannot intersect the hole (and generate more pieces) for the first $p_{0}$ iterates. Then from time $p_{0}$ to time $t_{1}$, it can be cut at most $1+\left[\left(t_{1}-p_{0}\right) /\right.$ $m_{0}$ ] times, where [.] denotes the greatest integer function. This will be true on each 
time interval $\left[t_{i}, t_{i+1}\right]$. Thus

$$
\begin{aligned}
\log _{2}\left(\# S_{n}\right) \leq & 1+\left[\frac{t_{1}-p_{0}}{m_{0}}\right]+1+\left[\frac{t_{2}-\left(t_{1}+p_{1}\right)}{m_{0}}\right]+\cdots \\
& +1+\left[\frac{t_{s}-\left(t_{s-1}+p_{s-1}\right)}{m_{0}}\right]+1+\left[\frac{n-\left(t_{s}+p_{s}\right)}{m_{0}}\right] \\
\leq & \begin{cases}s+1+\frac{n-\sum_{i=0}^{s} p_{i}}{m_{0}} & \text { if } n \geq t_{s}+p_{s} \\
s+\frac{t_{s}-\sum_{i=0}^{s-1} p_{i}}{m_{0}} & \text { if } n<t_{s}+p_{s}\end{cases} \\
\leq & s+1+\frac{n-\frac{1}{2} \sum_{i=0}^{s-1}\left|r_{i}\right|}{m_{0}},
\end{aligned}
$$

where we have used Property P1(a) in the last step. Now we are ready to estimate the tail of the return time function $S$.

We begin with an interval $\Omega$ which may or may not be a subset of $(-\delta, \delta)$. Suppose $\omega \in \Omega_{n}$ has itinerary $\left(r_{0}, \ldots, r_{s}\right)$ at times $t_{0}, \ldots, t_{s}$ with $s \geq 1$ and $t_{0}=0$. If $\omega \subset I_{k}$, let $r_{0}=k$; otherwise, let $r_{0}=0$. Assume $|k| \leq n / 8$. For $x \in \omega$,

$$
\left|\left(\hat{T}^{n}\right)^{\prime}(x)\right|=\left|\left(\hat{T}^{n-t_{s}}\right)^{\prime}\left(\hat{T}^{t_{s}} x\right)\right| \prod_{i=0}^{s-1}\left|\left(\hat{T}^{t_{i+1}-t_{i}}\right)^{\prime}\left(\hat{T}^{t_{i}} x\right)\right| .
$$

We estimate $\left|\left(\hat{T}^{t_{i+1}-t_{i}}\right)^{\prime}\left(\hat{T}^{t_{i}} x\right)\right|$ using a method similar to the proof of Lemma 2.5 . Let $s_{0}=t_{i}, s_{k+1}=t_{i+1}$ and $s_{1}, \ldots, s_{k}$ be the times when $\omega$ returns to $\left(-\delta_{0}, \delta_{0}\right)$ between times $t_{i}$ and $t_{i+1}$. On each interval $\left[s_{j}, s_{j+1}\right]$ (except possibly when $i=0$ and $j=0$ ), we are in Case 1 of the proof of Lemma 2.5 so $\left|\left(\hat{T}^{s_{j+1}-s_{j}}\right)^{\prime}\left(\hat{T}^{t_{i}+s_{j}} x\right)\right| \geq e^{\frac{1}{3} \lambda_{0}\left(s_{j+1}-s_{j}\right)}$. Stringing these intervals together, we have

$$
\left|\left(\hat{T}^{t_{i+1}-t_{i}}\right)^{\prime}\left(\hat{T}^{t_{i}} x\right)\right| \geq e^{\frac{1}{3} \lambda_{0}\left(t_{i+1}-t_{i}\right)}
$$

for $i>0$ and $\left|\left(\hat{T}^{t_{1}}\right)^{\prime}(x)\right| \geq c_{0} e^{\frac{1}{3} \lambda_{0} t_{1}}$ This yields

$$
\left|\left(\hat{T}^{n}\right)^{\prime}(x)\right| \geq\left|\left(\hat{T}^{n-t_{s}}\right)^{\prime}\left(\hat{T}^{t_{s}} x\right)\right| \cdot c_{0} e^{\frac{1}{3} \lambda_{0} t_{s}} .
$$

If $n \geq t_{s}+p_{s}$, then $\left|\left(\hat{T}^{n-t_{s}}\right)^{\prime}\left(\hat{T}^{t_{s}} x\right)\right| \geq c_{0}^{\prime} \delta e^{\frac{1}{3} \lambda_{0}\left(n-t_{s}-p_{s}\right)} e^{p_{s} / 5}$ using Property P1(c) and Lemma 2.5(a). Combining these estimates, we have

$$
\left|\left(\hat{T}^{n}\right)^{\prime}(x)\right| \geq c_{0} \cdot c_{0}^{\prime} \delta e^{\frac{1}{3} \lambda_{0} n} .
$$

Since $\left|\hat{T}^{n} \omega\right|<4^{8} \varepsilon$, we can estimate

$$
|\omega| \leq \frac{4^{8} \varepsilon}{\delta c_{0} c_{0}^{\prime} e^{\frac{1}{3} \lambda_{0} n}} \leq \frac{1}{c_{0} c_{0}^{\prime}} e^{-\frac{1}{3} \lambda_{0} n}
$$

If $n<t_{s}+p_{s}$ then we note that at time $t_{s},\left|\left(\hat{T}^{t_{s}}\right)^{\prime}(x)\right| \geq e^{\frac{1}{3} \lambda_{0} t_{s}}$. Since $T^{t_{s}} \omega \subset I_{r_{s}}$, we estimate

$$
|\omega| \leq \frac{e^{-\left|r_{s}\right|}}{c_{0} e^{\frac{1}{3} \lambda_{0} t_{s}}} \leq \frac{1}{c_{0}} e^{-\frac{1}{3} \lambda_{0}\left(t_{s}+4\left|r_{s}\right|\right)} e^{-\left|r_{s}\right| / 8} \leq \frac{1}{c_{0}} e^{-\frac{1}{3} \lambda_{0} n} e^{-\left|r_{s}\right| / 8} .
$$


We define $A\left(r_{1}, \ldots, r_{s}\right)=\left\{x \in \omega: S(x)>n, x\right.$ has itinerary $\left.r_{1}, \ldots, r_{s}\right\}$ and $K=\sum_{i=1}^{s}\left|r_{i}\right|$. For fixed $s$ and $K$, we set $A_{s, K}^{n}=\bigcup_{\substack{\left(r_{1}, \ldots, r_{s}\right) \\ r_{i} \mid=K}} A\left(r_{1}, \ldots r_{s}\right)$ and estimate

$$
\#\left\{s \text {-tuples with } \sum\left|r_{i}\right|=K\right\}<2^{s}\left(\begin{array}{c}
K-1 \\
s-1
\end{array}\right) .
$$

Note that since each $\left|r_{i}\right| \geq \Delta:=\log 1 / \delta$, we have $s \leq K / \Delta$. So

$$
\#\left\{s \text {-tuples with } \sum\left|r_{i}\right|=K\right\}<2^{s}\left(\begin{array}{c}
K-1 \\
K / \Delta-1
\end{array}\right)<2^{s}(1+\sigma(\delta))^{K},
$$

where $\sigma(\delta) \rightarrow 0$ as $\delta \rightarrow 0$. Now we estimate

$$
m\left(A_{s, K}^{n}\right) \leq\left(\begin{array}{c}
\# s-\text { tuples with } \\
\sum\left|r_{i}\right|=K
\end{array}\right) \cdot\left(\begin{array}{c}
\# \text { pieces with } \\
\text { itinerary }\left(r_{1}, \ldots, r_{s}\right)
\end{array}\right) \cdot m\left\{\begin{array}{c}
\text { one piece with } \\
\text { itinerary }\left(r_{1}, \ldots, r_{s}\right)
\end{array}\right\} .
$$

Let $\Omega^{\prime}$ be those points in $\Omega \cap I_{k}$ with $S(x)>n$ which have made at least one return to $(-\delta, \delta)$.

$$
m\left(\Omega^{\prime}\right) \leq \sum_{K \leq \frac{3}{2} n} \sum_{s=1}^{K / \Delta} m\left(A_{s, K}^{n}\right)+\sum_{K>\frac{3}{2} n} \sum_{s=1}^{K / \Delta} m\left(A_{s, K}^{n}\right) .
$$

For the first term, we use equation (4), the maximum of (7) and (8) and equation (9) to observe that

$$
m\left(A_{s, K}^{n}\right) \leq 2^{s}(1+\sigma(\delta))^{K} \cdot 2^{s+1} 2^{n / m_{0}} \cdot \frac{1}{c_{0} c_{0}^{\prime}} e^{-\frac{1}{3} \lambda_{0} n} .
$$

The competing factors in this expression are $e^{-\frac{1}{3} \lambda_{0} n}$ and $2^{n / m_{0}}$. Taking $\lambda_{0}=\log 1.9$ and $m_{0} \geq 10$, we estimate their product by $e^{n\left(\left(\log 2 / m_{0}\right)-\left(\lambda_{0} / 3\right)\right)} \leq e^{-\frac{1}{7} n}$.

Since $n \geq \frac{2}{3} K$ and $n \geq 8|k|=8\left|r_{0}\right|$, we may write $n \geq n / 3+K / 3+8\left|r_{0}\right| / 6$. Equation (11) becomes

$$
m\left(A_{s, K}^{n}\right) \leq 2(1+\sigma(\delta))^{K} 4^{s} \frac{1}{c_{0} c_{0}^{\prime}} e^{-\frac{1}{21} n} e^{-\frac{1}{21} K} e^{-\frac{4}{21}\left|r_{0}\right|} .
$$

To estimate the second term of equation (10), we note that $t_{s}-\sum_{i=0}^{s-1} p_{i} \leq n-$ $\frac{1}{2} \sum_{i=0}^{s-1}\left|r_{i}\right|=n-K / 2+\left|r_{s}\right| / 2$. Applying this observation to equation (4) and using equation (8) we estimate

$$
m\left(A_{s, K}^{n}\right) \leq 2^{s}(1+\sigma(\delta))^{K} 2^{s+1} 2^{(n-K / 2) / m_{0}} 2^{\left|r_{s}\right| / 2 m_{0}} \frac{1}{c_{0}} e^{-\frac{1}{3} \lambda_{0}\left(t_{s}+4\left|r_{s}\right|\right)} e^{-\left|r_{s}\right| / 8} .
$$

The competing factors in this expression are $e^{-\frac{1}{3} \lambda_{0}\left(t_{s}+4\left|r_{s}\right|\right)}$ and $2^{(n-K / 2) / m_{0}}$. Using the fact that $n \leq \frac{2}{3} K$ and $t_{s} \geq \frac{1}{2} \sum_{i=0}^{s-1}\left|r_{i}\right|$, these terms become $e^{-\frac{1}{3} \lambda_{0}(K / 2)} e^{-\frac{1}{3} \lambda_{0}\left(\left|r_{0}\right| / 2\right)}$ and $2^{K / 6 m_{0}}$. Then if $m_{0} \geq 10$ we conclude

$$
m\left(A_{s, K}^{n}\right) \leq 2(1+\sigma(\delta))^{K} 4^{s} \frac{1}{c_{0}} e^{-K / 11} e^{-\frac{1}{6} \lambda_{0}\left|r_{0}\right|} .
$$


Substituting equations (12) and (14) into equation (10) and summing over $s$, we get

$$
\begin{aligned}
m\left(\Omega^{\prime}\right) \leq & \sum_{K \leq \frac{3}{2} n} 2(1+\sigma(\delta))^{K} 4^{(K / \Delta)+1} \frac{1}{c_{0} c_{0}^{\prime}} e^{-\frac{1}{21} K} e^{-\frac{1}{21} n} e^{-\frac{4}{21}\left|r_{0}\right|} \\
& +\sum_{K>\frac{3}{2} n} 2(1+\sigma(\delta))^{K} 4^{(K / \Delta)+1} \frac{1}{c_{0}} e^{-K / 11} e^{-\frac{1}{6} \lambda_{0}\left|r_{0}\right|} \\
\leq & c_{4} e^{-\frac{1}{21} n} e^{-\frac{4}{21}\left|r_{0}\right|}+\sum_{K>\frac{3}{2} n} c_{5} e^{-K / 30} e^{-\frac{1}{6} \lambda_{0}\left|r_{0}\right|} \\
\leq & c_{6} e^{-n / 21} e^{-\frac{1}{6} \lambda_{0}\left|r_{0}\right|}
\end{aligned}
$$

if $\delta$ is taken to be sufficiently small. Note that $\Delta>50$ is forced by these estimates.

Let $\Omega^{\prime \prime}$ be the set of points in $\Omega \cap I_{k}$ which have $S(x)>n$ and which have never returned to $(-\delta, \delta)$. In this case, we have a simple estimate using Lemma 2.5(a) that $\left|\left(\hat{T}^{n}\right)^{\prime}(x)\right| \geq c_{0}^{\prime} \delta e^{\frac{1}{3} \lambda_{0} n}$. Since we are assuming that $|k| \leq n / 8$, we have $p_{0} \leq 4|k| \leq n / 2$ so using equation (4), the estimate on the number of pieces which can be formed up to time $n$, we have

$$
m\left(\Omega^{\prime \prime}\right) \leq 2^{\left(n-p_{0}\right) / m_{0}} \frac{4^{8} \varepsilon}{c_{0}^{\prime} \delta} e^{-\frac{1}{3} \lambda_{0} n} \leq \frac{1}{c_{0}^{\prime}} 2^{n / m_{0}} e^{-\frac{1}{3} \lambda_{0} \frac{7}{8} n} e^{-\frac{1}{3} \lambda_{0}|k|} \leq \frac{1}{c_{0}^{\prime}} e^{-n / 9} e^{-\frac{1}{3} \lambda_{0}\left|r_{0}\right|} .
$$

Now if $\Omega$ is not a subset of $(-\delta, \delta)$, then $r_{0}=0$ and we have shown that

$$
m\{x \in \Omega: S(x)>n\}=m\left(\Omega^{\prime}\right)+m\left(\Omega^{\prime \prime}\right) \leq\left(c_{6}+\frac{1}{c_{0}^{\prime}}\right) e^{-n / 21} .
$$

On the other hand, if $\Omega \subset(-\delta, \delta)$, then

$$
\begin{aligned}
m\{x \in \Omega: S(x)>n\} & \leq \sum_{|k| \leq n / 8}\left(c_{6}+\frac{1}{c_{0}^{\prime}}\right) e^{-n / 21} e^{-\frac{1}{6} \lambda_{0}|k|}+m\left(\Omega \cap\left(-e^{-n / 8}, e^{-n / 8}\right)\right) \\
& \leq\left(c_{6}+\frac{1}{c_{0}^{\prime}}\right) e^{-n / 21} \sum_{k \geq 1} e^{-\frac{1}{6} \lambda_{0} k}+e^{-n / 8} \\
& \leq c_{7} e^{-n / 21}
\end{aligned}
$$

which proves Proposition 4.1(e) with $C^{\prime}=\max \left\{c_{7}, c_{6}+1 / c_{0}^{\prime}\right\}$.

4.3. Assembling the complete tower. We now have the required tools to complete the construction of the tower. This construction is achieved by applying Proposition 4.1 repeatedly to the reference intervals $\Lambda^{(i)}$ introduced at the beginning of $\S 4$. We fix $j$ and proceed one $\Lambda^{(j)}$ at a time. Our construction will result in a partition and stopping time with the following properties.

Proposition 4.2. There exists a countable partition $\eta$ of $\Lambda^{(j)}$ and a stopping time $R$ satisfying:

(a) $R$ is constant on each element $\omega \in \eta$;

(b) either $T^{R} \omega$ is defined and $T^{R} \omega=\Lambda^{(i)}$ for some $i$, or $T^{R-1} \omega$ is defined and $\hat{T}^{R} \omega \subset H$; 
(c) for $x, y \in \omega$,

$$
\left|\frac{\left(\hat{T}^{R}\right)^{\prime}(x)}{\left(\hat{T}^{R}\right)^{\prime}(y)}-1\right| \leq \tilde{C}\left|\hat{T}^{R}(x)-\hat{T}^{R}(y)\right|
$$

(d) $\left|\left(T^{R}\right)^{\prime}(x)\right|>4^{6}$

(e) $m\left\{x \in \Lambda^{(j)}: R(x)>n\right\} \leq C^{\prime \prime} \theta^{n}$ for some $\theta<1$ and $C^{\prime \prime}$ independent of $\delta$.

Proof. Since $\Lambda^{(j)}$ is an interval of the form $\Omega$ in Proposition 4.1, there exists a partition $\mathcal{Z}_{1}$ of $\Lambda^{(j)}$ and a stopping time $S_{1}$ with the properties of that proposition. For each $\omega_{1} \in \mathcal{Z}_{1}$, we do the following.

Case 1. $\hat{T}^{S_{1}} \omega_{1} \in H$. We set $R(x)=S_{1}(x)$ for $x \in \omega_{1}$ and enter $\omega_{1}$ as an element of the partition $\eta$.

Case 2. $\left|\hat{T}^{S_{1}} \omega_{1}\right| \geq 4^{8} \varepsilon$. In this case, $\hat{T}^{S_{1}} \omega_{1}$ must completely cover at least $4^{7}$ of the $\Lambda^{(i)}$, plus at most one extra piece on each side. If the left-most end piece has length less than $\varepsilon$, then we adjoin it to the left-most $\Lambda^{(i)}$ that is covered by $\hat{T}^{S_{1}} \omega_{1}$; otherwise we leave it. We do the same for the right end piece. For each of the $\Lambda^{(i)}$ that has not been adjoined to the end pieces, we enter $\omega_{1} \cap T^{-S_{1}} \Lambda^{(i)}$ as an element of $\eta$ and declare $R(x)=S_{1}(x)$ on this interval.

For each $\omega_{1} \in \mathcal{Z}_{1}$ with $\left|\hat{T}^{S_{1}} \omega_{1}\right| \geq 4^{8} \varepsilon$, we are left with at most two pieces $\omega_{1}^{ \pm}$with $\varepsilon \leq\left|\hat{T}^{S_{1}} \omega_{1}^{ \pm}\right| \leq 3 \varepsilon$ on which $R$ has not yet been declared. We apply Proposition 4.1 to obtain a partition and stopping time $S$ on each interval $\hat{T}^{S_{1}} \omega_{1}^{ \pm}$. Define $S_{2}=S_{1}+S \circ \hat{T}^{S_{1}}$ on $\omega_{1}^{ \pm}$. This induces a partition $\mathcal{Z}_{2}$ on $\Lambda^{(j)} \backslash\left\{x: R(x)=S_{1}(x)\right\}$. For each piece $\omega_{2} \in \mathcal{Z}_{2}$ we apply Cases 1 and 2 described above and as before, are left with two pieces $\hat{T}^{S_{2}} \omega_{2}^{ \pm}$ each with length between $\varepsilon$ and $3 \varepsilon$ such that $R$ has not yet been declared on $\omega_{2}^{ \pm}$. We use Proposition 4.1 to define $S_{3}=S_{2}+S \circ \hat{T}^{S_{2}}$ on $\Lambda^{(j)} \backslash\left(\left\{R=S_{1}\right\} \cup\left\{R=S_{2}\right\}\right)$ and proceed inductively.

Continuing in this way, we generate a sequence of stopping times $S_{i}$ and partitions $\mathcal{Z}_{i}$ of $\Lambda^{(j)} \backslash\left(\cup_{k=1}^{i}\left\{R=S_{k}\right\}\right)$ such that $S_{k}$ is constant on $\omega_{i} \in \mathcal{Z}_{i}$ for each $k \leq i$. It is clear that $R$ and $\eta$ as constructed above satisfy items (a)-(d) by Proposition 4.1. We now derive the tail estimate (e).

Fix an $\omega_{i} \in \mathcal{Z}_{i}$ and let $\varphi_{i}$ denote the inverse of $\hat{T}^{S_{i}}$ restricted to $\omega_{i}$. Then part (e) of Proposition 4.1 yields

$$
m\left\{x \in \hat{T}^{S_{i}} \omega_{i}^{ \pm}: S_{i+1}\left(\varphi_{i} x\right)-S_{i}\left(\varphi_{i} x\right)>n\right\} \leq C^{\prime} e^{-n / 21}
$$

Using the distortion bound of Lemma 4.6, this becomes

$$
m\left\{x \in \omega_{i}^{ \pm}: S_{i+1}(x)-S_{i}(x)>n\right\} \leq e C^{\prime} \frac{\left|\omega_{i}^{ \pm}\right|}{\left|\hat{T}^{S_{i}} \omega_{i}^{ \pm}\right|} e^{-n / 21} \leq e C^{\prime} \frac{\left|\omega_{i}^{ \pm}\right|}{\varepsilon} e^{-n / 21} .
$$

Since we return at least $1-6 / 4^{8}$ of $\hat{T}^{S_{i+1}} \omega_{i}^{ \pm}$, we conclude, again using distortion bounds, that at least $\frac{1}{3}$ of $\omega_{i}^{ \pm}$is returned at time $S_{i+1}$, i.e.

$$
m\left\{x \in \omega_{i}^{ \pm}: R(x)=S_{i+1}(x)\right\} \geq \frac{\left|\omega_{i}^{ \pm}\right|}{3} .
$$


We wish to estimate $m\left\{x \in \Lambda^{(j)}: R(x)>n\right\}$. Let $\alpha>0$ be a small number to be chosen later. So

$$
m\{R(x)>n\}=\sum_{i \leq[\alpha n]} m\left\{S_{i-1} \leq n<S_{i}\right\}+\sum_{i>[\alpha n]} m\left\{S_{i-1} \leq n<S_{i}\right\} .
$$

Using equation (16) and summing over pieces $\omega_{i}^{ \pm}$in $\Lambda^{(j)}$, the second sum can be estimated by

$$
\sum_{i>[\alpha n]} m\left\{S_{i-1} \leq n<S_{i} \leq R(x)\right\} \leq \sum_{i>[\alpha n]}\left(\frac{2}{3}\right)^{i-1}\left|\Lambda^{(j)}\right|=\left(\frac{2}{3}\right)^{[\alpha n]} 3\left|\Lambda^{(j)}\right| .
$$

To estimate the first sum in equation (17), we define $A_{i}\left(l_{1}, \ldots, l_{i-1}\right)=\left\{x \in \Lambda^{(j)}\right.$ : $R(x)>n, S_{i-1} \leq n<S_{i}$ and $\left.S_{k}-S_{k-1}=l_{k}, k=1, \ldots, i-1\right\}$. Each term in the first sum can be estimated by

$$
m\left\{R(x)>n: S_{i-1} \leq n<S_{i}\right\}=\sum_{\substack{\left(l_{1}, \ldots, l_{i-1}\right) \\ \sum_{k} l_{k} \leq n}} m\left(A_{i}\left(l_{1}, \ldots, l_{i-1}\right)\right) .
$$

For a fixed $\left(l_{1}, \ldots, l_{i-1}\right)$ let $B_{m}=\left\{x \in \Lambda^{(j)}: R(x)>S_{m}(x)\right.$ and $S_{k}-S_{k-1}=l_{k}, k=$ $1, \ldots, m\}$. We condition on the $B_{m}$ to obtain

$$
m\left(A_{i}\left(l_{1}, \ldots, l_{i-1}\right)\right)=m\left\{R>n \geq S_{i-1} \mid B_{i-1}\right\} \prod_{m=1}^{i-1} m\left\{S_{m}-S_{m-1}=l_{m} \mid B_{m-1}\right\} .
$$

We estimate this product using equation (15):

$$
\begin{aligned}
m\left(A_{i}\left(l_{1}, \ldots, l_{i-1}\right)\right) & \leq e C^{\prime} \frac{\left|\Lambda^{(j)}\right|}{\varepsilon} e^{-\left(n-\sum_{k} l_{k}\right) / 21} \prod_{k=1}^{i-1} e C^{\prime} \frac{\left|\Lambda^{(j)}\right|}{\varepsilon} e^{-\left(l_{k}-1\right) / 21} \\
& \leq\left(2 e C^{\prime}\right)^{i} e^{i / 21} e^{-n / 21} .
\end{aligned}
$$

Now we estimate

$$
\#\left\{\left(l_{1}, \ldots, l_{i-1}\right): \sum_{k} l_{k} \leq n\right\} \leq\left(\begin{array}{c}
n \\
i-1
\end{array}\right) \leq(1+\tau(\alpha))^{n},
$$

where $\tau(\alpha) \rightarrow 0$ as $\alpha \rightarrow 0$. Using equations (18)-(20), equation (17) becomes

$$
\begin{aligned}
m\{R(x)>n\} & \leq \sum_{i \leq[\alpha n]}(1+\tau(\alpha))^{n}\left(2 e C^{\prime}\right)^{i} e^{i / 21} e^{-n / 21}+\left(\frac{2}{3}\right)^{[\alpha n]} 3\left|\Lambda^{(j)}\right| \\
& \leq(1+\tau(\alpha))^{n}\left(2 e C^{\prime}\right)^{\alpha n+1} e^{(\alpha n+1) / 21} e^{-n / 21}+\left(\frac{2}{3}\right)^{[\alpha n]} 3\left|\Lambda^{(j)}\right| \\
& \leq C^{\prime \prime} \theta^{n},
\end{aligned}
$$

where $\theta:=\left(\frac{2}{3}\right)^{\alpha}$ for the optimal $\alpha$ which makes $\left(\frac{2}{3}\right)^{\alpha}=(1+\tau(\alpha))\left(2 e C^{\prime}\right)^{\alpha} e^{(\alpha-1) / 21}$. 
4.4. Estimating the amount that falls in the hole. For each $\Lambda^{(j)}$, we estimate the amount of Lebesgue measure that can fall into the hole $H$ at a given time $n$. This estimate will resemble the estimates of $\S \S 4.2$ and 4.3. We prove the following proposition.

Proposition 4.3. There exists a $D>0$ such that for any $\Lambda^{(j)}$,

$$
m\left\{x \in \Lambda^{(j)}: R(x)=n \text { and } \hat{T}^{n} x \in H\right\} \leq D m(H) \theta^{n} .
$$

The proof of this proposition will depend on the following lemma in much the same way that the proof of Proposition 4.2 used Proposition 4.1.

Lemma 4.4. Let $\Omega, \mathcal{Z}$ and $S$ be as in the statement of Proposition 4.1. There exists a $D^{\prime}>0$ such that

$$
m\left\{x \in \Omega: S(x)=n \text { and } \hat{T}^{n} x \in H\right\} \leq D^{\prime} m(H) e^{-n / 21} .
$$

Proof. Suppose $\omega \in \mathcal{Z}$ and $\hat{T}^{S} \omega \subset H$ with $S=n$. If $\omega \subset I_{k}$, we set $r_{0}=k$ and $p_{0}=p\left(r_{0}\right)$; otherwise, set $r_{0}=p_{0}=0$. Suppose $\omega$ has itinerary $\left(r_{1}, \ldots, r_{s}\right)$ at times $t_{1}, \ldots, t_{s}$ and let $\left(p_{1}, \ldots, p_{s}\right)$ be the associated recovery times. Note that since $\hat{T}^{n} \omega \subset H$, $\omega$ must be free at time $n$ so that $n \geq t_{s}+p_{s}$. Using equation (6), we obtain

$$
|\omega| \leq \frac{m H}{c_{0} c_{0}^{\prime} \delta} e^{-\frac{1}{3} \lambda_{0} n}
$$

Since $\hat{T}^{n} \omega$ is free, we have $n \geq p_{0}+\sum_{i=1}^{s} p_{i} \geq \frac{1}{2}\left(\left|r_{0}\right|+\sum_{i=1}^{s}\left|r_{i}\right|\right)$ and $s \leq K / \Delta$, where $K=\sum_{i=1}^{s}\left|r_{i}\right|$. We estimate using equations (3) and (21):

$$
\begin{aligned}
m\left\{x \in I_{r_{0}}: \hat{T}^{n} x \in H\right\} & \leq \sum_{K=0}^{2 n} \sum_{s=0}^{K / \Delta}\left(\begin{array}{c}
\# s \text {-tuples with } \\
\sum\left|r_{i}\right|=K
\end{array}\right) \cdot\left(\begin{array}{c}
\text { \# pieces with } \\
\text { same itinerary }
\end{array}\right) \cdot\left(\begin{array}{c}
\text { measure } \\
1 \text { piece }
\end{array}\right) \\
& \leq \sum_{K=0}^{2 n} \sum_{s=0}^{K / \Delta} 2^{s}\left(\begin{array}{c}
K-1 \\
s-1
\end{array}\right) \cdot 2^{s+1} 2^{(n-(K / 2)) / m_{0}} \cdot \frac{m H}{c_{0} c_{0}^{\prime} \delta} e^{-\frac{1}{3} \lambda_{0} n} \\
& \leq \sum_{K=0}^{2 n} 2(1+\sigma(\delta))^{K} 4^{(K / \Delta)+1} 2^{-K / 2 m_{0}} 2^{n / m_{0}} \frac{m H}{c_{0} c_{0}^{\prime} \delta} e^{-\frac{1}{3} \lambda_{0} n} \\
& \leq \frac{m H}{c_{0} c_{0}^{\prime} \delta} 8(1+\sigma(\delta))^{2 n+1} 2^{n / m_{0}} e^{-\frac{1}{4} \lambda_{0} n} e^{-\frac{1}{12} \lambda_{0}\left|r_{0}\right|} \\
& \leq c_{8} m(H) e^{-\frac{1}{21} n} e^{-\frac{1}{12} \lambda_{0}\left|r_{0}\right|}
\end{aligned}
$$

for $\delta$ small enough and $m_{0}=10$. But since $\delta$ has already been chosen small enough to make a comparable series converge in $\S 4.2$, the same $\delta$ will work here.

If $\Omega \subset I \backslash(-\delta, \delta)$, then $r_{0}=0$ and the above estimate shows that

$$
m\left\{x \in \Omega: S(x)=n \text { and } \hat{T}^{n} x \in H\right\} \leq c_{8} m(H) e^{-\frac{1}{21} n} .
$$

If $\Omega \subset(-\delta, \delta)$, then summing across the $I_{k}$, we have

$$
m\left\{x \in \Omega: S(x)=n \text { and } \hat{T}^{n} x \in H\right\} \leq c_{8} m(H) e^{-\frac{1}{21} n} \sum_{k=k_{0}}^{\infty} e^{-\frac{1}{12} \lambda_{0} k} .
$$


This proves the lemma with

$$
D^{\prime}:=\max \left\{c_{8}, c_{8} \frac{e^{-\frac{1}{12} \lambda_{0} k_{0}}}{1-e^{-\frac{1}{12} \lambda_{0}}}\right\} .
$$

Note that $D^{\prime} \sim 1 / \delta$.

Proof of Proposition 4.3. Let $F_{n}=\left\{x \in \Lambda^{(j)}: R(x)=n\right.$ and $\left.\hat{T}^{n} x \in H\right\}$. Each $x \in F_{n}$ will have a number of auxiliary stopping times defined by the construction described in $\S 4.3$. We define $S_{*}(x)$ to be the time when $x$ starts its final auxiliary stopping time before falling into the hole. Let $F_{n}^{i}=\left\{x \in F_{n}: S_{*}(x)=i\right\}$. Note that if $x \in F_{n}^{i}$ then $x \in \omega_{*}^{ \pm}$and $T^{S_{*}(x)} \omega_{*}^{ \pm}$is an interval of the type $\Omega$ in Lemma 4.4. Thus

$$
m\left\{y \in T^{S_{*}(x)} \omega_{*}^{ \pm}: S(y)=k \text { and } \hat{T}^{k}(y) \in H\right\} \leq D^{\prime} m(H) e^{-k / 21} .
$$

Using the distortion bound of Lemma 4.6, we obtain

$$
\begin{aligned}
m\left\{x \in \omega_{*}^{ \pm}: \hat{T}^{n}(x) \in H\right\} & \leq e D^{\prime} m H \frac{\left|\omega_{*}^{ \pm}\right|}{\left|T^{S_{*}(x)} \omega_{*}^{ \pm}\right|} e^{-\left(n-S_{*}(x)\right) / 21} \\
& \leq e D^{\prime} m H \frac{\left|\omega_{*}^{ \pm}\right|}{\varepsilon} e^{-(n-i) / 21} .
\end{aligned}
$$

Using equation (22) and Proposition 4.2, we estimate the size of $F_{n}$ :

$$
\begin{aligned}
m\left(F_{n}\right) & =\sum_{i=0}^{n-1} m\left(F_{n}^{i}\right)=\sum_{i=0}^{n-1} m\left\{\hat{T}^{n} \in H \mid S_{*}(x)=i\right\} \cdot m\left\{S_{*}(x)=i\right\} \\
& \leq \sum_{i=0}^{n-1} 2 e D^{\prime} m(H) e^{-(n-i) / 21} \cdot C^{\prime \prime} \theta^{i} \leq 2 e D^{\prime} C^{\prime \prime} m(H) \theta^{n} \sum_{i=1}^{\infty} \frac{1}{\left(e^{\frac{1}{21}} \theta\right)^{i}} \\
& =: \operatorname{Dm}(H) \theta^{n} .
\end{aligned}
$$

Note again that $D \sim 1 / \delta$.

4.5. Distortion bounds. We begin this section by deriving the distortion bound on which Properties P1 are based. The content of this estimate is essentially to show that the derivative $\left(\hat{T}^{n}\right)^{\prime}(\hat{T} x)$ for $x \in(-\delta, \delta)$ is comparable to $\left(\hat{T}^{n}\right)^{\prime}(\hat{T} 0)$ for $0 \leq n \leq p(x)-1$ and so grows exponentially. We estimate

$$
\begin{aligned}
\log \frac{\left(\hat{T}^{n-1}\right)^{\prime}(\hat{T} x)}{\left(\hat{T}^{n-1}\right)^{\prime}(\hat{T} 0)} & \leq \sum_{j=1}^{n-1}|\log | \hat{T}^{j}(x)|-\log | \hat{T}^{j}(0)|| \\
& \leq \frac{1}{\delta_{0}} \sum_{j=1}^{n-1}\left|\hat{T}^{j}(x)-\hat{T}^{j}(0)\right| \\
& \leq \frac{1}{\delta_{0}} \sum_{j=1}^{n-1} \frac{1}{j^{2}} .
\end{aligned}
$$

So we have

$$
d_{0}^{-1}(1.9)^{n} \leq\left|\left(\hat{T}^{n}\right)^{\prime}(\hat{T} x)\right| \leq d_{0}(1.9)^{n}
$$


for some $d_{0}>1$ for each $0 \leq n \leq p(x)-1$. We will use this estimate in $\S 5.4$ when determining an upper bound for the conditionally invariant density. The remaining results in this section concern the time $q(k)$ and the main distortion bound for pieces which return at times $S$ and $R$.

Condition A1 implies that for each $k \geq k_{0}$ the interval $\left(0, e^{-k}\right)$ must grow to at least length $r$ before intersecting $H$. Recall $q(k)$ as defined in $\$ 2.4$. The following lemma allows us to conclude that each $I_{k}$ must grow to a fixed length by time $q(k)$.

LEMmA 4.5. There exists a $c_{1}>0$ independent of $\delta$ such that for any $k$ with $k \geq k_{0}$ and any $n$ with $M_{0}<n \leq q(k)$ :

(a) if $x, y \in\left(0, e^{-k}\right)$, then

$$
\log \frac{\left(\hat{T}^{n-1}\right)^{\prime}(\hat{T} x)}{\left(\hat{T}^{n-1}\right)^{\prime}(\hat{T} y)} \leq c_{1}
$$

(b) if $x, y \in I_{k}$, then

$$
\log \frac{\left(\hat{T}^{n}\right)^{\prime}(x)}{\left(\hat{T}^{n}\right)^{\prime}(y)} \leq c_{1}
$$

Proof. Write $n-1=l M_{0}+j$ for some $l \in \mathbb{Z}^{+}, 0 \leq j \leq M_{0}-1$. For $x, y \in I_{k}$, we estimate the derivatives using Definition 2.1(b)(i):

$$
\begin{aligned}
& \log \frac{\left(\hat{T}^{n}\right)^{\prime}(x)}{\left(\hat{T}^{n}\right)^{\prime}(y)} \leq\left|\log \frac{x}{y}\right|+\sum_{i=0}^{l-2}|\log |\left(\hat{T}^{M_{0}}\right)^{\prime}\left(\hat{T}^{1+i M_{0}} x\right)|-\log |\left(\hat{T}^{M_{0}}\right)^{\prime}\left(\hat{T}^{1+i M_{0}} y\right)|| \\
& +|\log |\left(\hat{T}^{M_{0}+j}\right)^{\prime}\left(\hat{T}^{n-\left(M_{0}+j\right)} x\right)|-\log |\left(\hat{T}^{M_{0}+j}\right)^{\prime}\left(\hat{T}^{n-\left(M_{0}+j\right)} y\right)|| \\
& \leq\left|\log \frac{x}{y}\right|+\sum_{i=0}^{l-2} \frac{1}{e^{\lambda_{0} M_{0}}}\left|\left(\hat{T}^{M_{0}}\right)^{\prime}\left(\hat{T}^{1+i M_{0}} x\right)-\left(\hat{T}^{M_{0}}\right)^{\prime}\left(\hat{T}^{1+i M_{0}} y\right)\right| \\
& +\frac{1}{e^{\lambda_{0}\left(M_{0}+j\right)}}\left|\left(\hat{T}^{M_{0}+j}\right)^{\prime}\left(\hat{T}^{n-M_{0}-j} x\right)\right|-\left|\left(\hat{T}^{M_{0}+j}\right)^{\prime}\left(\hat{T}^{n-M_{0}-j} y\right)\right| \\
& \leq\left|\log \frac{x}{y}\right|+\sum_{i=0}^{l-2} \frac{(2 a)^{M_{0}}}{e^{\lambda_{0} M_{0}}}\left|\hat{T}^{1+i M_{0}}(x)-\hat{T}^{1+i M_{0}}(y)\right| \\
& +\frac{(2 a)^{M_{0}+j}}{e^{\lambda_{0}\left(M_{0}+j\right)}}\left|\hat{T}^{n-M_{0}-j}(x)-\hat{T}^{n-M_{0}-j}(y)\right| \\
& \leq\left|\log \frac{x}{y}\right|+\frac{(2 a)^{M_{0}}}{e^{\lambda_{0} M_{0}}} \sum_{i=0}^{l-2}\left|\hat{T}^{n}(x)-\hat{T}^{n}(y)\right| e^{-\lambda_{0}\left((l-i) M_{0}+j\right)} \\
& +\frac{(2 a)^{M_{0}+j}}{e^{\lambda_{0}\left(M_{0}+j\right)}}\left|\hat{T}^{n}(x)-\hat{T}^{n}(y)\right| e^{-\lambda_{0}\left(M_{0}+j\right)} \\
& \leq\left|\log \frac{x}{y}\right|+\left|\hat{T}^{n}(x)-\hat{T}^{n}(y)\right| \frac{(2 a)^{M_{0}}}{e^{\lambda_{0} M_{0}}} \sum_{i=2}^{\infty} e^{-\lambda_{0} M_{0} i} \\
& +\left|\hat{T}^{n}(x)-\hat{T}^{n}(y)\right| \frac{(2 a)^{2 M_{0}-1}}{e^{2 \lambda_{0}\left(2 M_{0}-1\right)}}
\end{aligned}
$$




$$
\begin{aligned}
& \leq 1+\frac{\delta_{0}}{2}\left(\frac{(2 a)^{M_{0}}}{e^{\lambda_{0} M_{0}}} \sum_{i=2}^{\infty} e^{-\lambda_{0} M_{0} i}+\frac{(2 a)^{2 M_{0}-1}}{e^{2 \lambda_{0}\left(2 M_{0}-1\right)}}\right) \\
& =: c_{1} .
\end{aligned}
$$

This proves (b) directly and (a) follows simply by omitting the first term of the sum.

Lemma 4.5 (a) says that for any $k \geq k_{0}$, the relative scale of the partition $\left\{\hat{T}\left(I_{j}\right)\right\}_{j \geq k}$ of $\hat{T}\left(\left(0, e^{-k}\right)\right)$ is maintained until time $q(k)$. The same is also true for $\hat{T}\left(\left(-e^{-k}, 0\right)\right)$. Since the ratio of $\left|\hat{T}\left(\left(0, e^{-k}\right)\right)\right|$ to $\left|\hat{T}\left(\left(0, e^{-k-1}\right)\right)\right|$ is $e^{2}$, the ratio of $\left|\hat{T}^{q}\left(\left(0, e^{-k}\right)\right)\right|$ to $\left|\hat{T}^{q}\left(\left(0, e^{-k-1}\right)\right)\right|$ is about $e^{2}$ as well. Also $\left|\hat{T}^{q}\left(\left(0, e^{-k}\right)\right)\right| \geq r / 8$ implies that $\left|\hat{T}^{q}\left(I_{k}\right)\right|$ is uniformly bounded below. This minimum length is the quantity $\varepsilon^{\prime}$ introduced in $\$ 2.4$.

Lemma 4.5(b) yields a distortion bound for $x, y \in I_{k}$ at time $q(k)$ :

$$
\frac{\left|\hat{T}^{q}(x)-\hat{T}^{q}(y)\right|}{|x-y|} \geq e^{-c_{1}} \frac{\left|\hat{T}^{q} I_{k}\right|}{\left|I_{k}\right|} \geq e^{-c_{1}} \frac{\varepsilon^{\prime}}{\left|I_{k}\right|} .
$$

This implies that

$$
|x-y| \leq e^{c_{1}} \frac{\left|I_{k}\right|}{\varepsilon^{\prime}}\left|\hat{T}^{q}(x)-\hat{T}^{q}(y)\right| .
$$

Now we substitute the above estimate into equation (24) to obtain

$$
\begin{aligned}
\log \frac{\left(\hat{T}^{q}\right)^{\prime}(x)}{\left(\hat{T}^{q}\right)^{\prime}(y)} & \leq \frac{|x-y|}{e^{-k-1}}+\left|\hat{T}^{q} x-\hat{T}^{q} y\right|\left(\frac{(2 a)^{M_{0}}}{e^{\lambda_{0} M_{0}}} \sum_{i=2}^{\infty} e^{-\lambda_{0} M_{0} i}+\frac{(2 a)^{2 M_{0}-1}}{e^{2 \lambda_{0}\left(2 M_{0}-1\right)}}\right) \\
& \leq\left|\hat{T}^{q} x-\hat{T}^{q} y\right|\left(e^{c_{1}} \frac{2}{\varepsilon^{\prime}}+\frac{(2 a)^{M_{0}}}{e^{\lambda_{0} M_{0}}} \sum_{i=2}^{\infty} e^{-\lambda_{0} M_{0} i}+\frac{(2 a)^{2 M_{0}-1}}{e^{2 \lambda_{0}\left(2 M_{0}-1\right)}}\right) \\
& =: c_{2}\left|\hat{T}^{q} x-\hat{T}^{q} y\right| .
\end{aligned}
$$

From this we conclude that for $x, y \in I_{k}$,

$$
\left|\frac{\left(\hat{T}^{q}\right)^{\prime}(x)}{\left(\hat{T}^{q}\right)^{\prime}(y)}-1\right| \leq c_{2}\left|\hat{T}^{q}(x)-\hat{T}^{q}(y)\right| .
$$

Equation (25) allows us to prove our main distortion lemma for an interval $\omega$ which is returned at time $n$.

LEMMA 4.6. (Distortion bounds) If an interval $\omega \subset I$ is such that $\hat{T} \omega$ lies in one element of $\mathcal{Q}$ for each $i \in[0, n]$ and $S(\omega)=n$, then there exists a constant $\tilde{C}>0$ such that

$$
\left|\frac{\left(\hat{T}^{n}\right)^{\prime}(x)}{\left(\hat{T}^{n}\right)^{\prime}(y)}-1\right| \leq \tilde{C}\left|\hat{T}^{n}(x)-\hat{T}^{n}(y)\right| .
$$

Proof. Let $t_{0}=0, t_{s+1}=n$ and $t_{1}, \ldots, t_{s}$ be the times that $\omega$ visits $(-\delta, \delta)$ before time $n$. $\hat{T}^{t_{i}} \omega \subset I_{k_{i}}$ so we set $q_{i}=q\left(k_{i}\right)$. Since $S(\omega)=n$, we know that $n>t_{s}+q_{s}$ so for each $i$ 
we can write $t_{i+1}=t_{i}+q_{i}+l_{i}$ for some $l_{i} \in \mathbb{N}$. Then using equation (25), we estimate

$$
\begin{aligned}
\log \frac{\left(\hat{T}^{n}\right)^{\prime}(x)}{\left(\hat{T}^{n}\right)^{\prime}(y)} & =\log \prod_{i=0}^{s} \frac{\left(\hat{T}^{q_{i}}\right)^{\prime}\left(\hat{T}^{t_{i}} x\right) \cdot\left(\hat{T}^{l_{i}}\right)^{\prime}\left(\hat{T}^{t_{i}+q_{i}} x\right)}{\left(\hat{T}^{q_{i}}\right)^{\prime}\left(\hat{T}^{t_{i}} y\right) \cdot\left(\hat{T}^{l_{i}}\right)^{\prime}\left(\hat{T}^{t_{i}+q_{i}} y\right)} \\
& =\sum_{i=0}^{s} \log \frac{\left(\hat{T}^{q_{i}}\right)^{\prime}\left(\hat{T}^{t_{i}} x\right)}{\left(\hat{T}^{q_{i}}\right)^{\prime}\left(\hat{T}^{t_{i}} y\right)}+\log \frac{\left(\hat{T}^{l_{i}}\right)^{\prime}\left(\hat{T}^{t_{i}+q_{i}} x\right)}{\left(\hat{T}^{l_{i}}\right)^{\prime}\left(\hat{T}^{t_{i}+q_{i}} y\right)} \\
& \leq \sum_{i=0}^{s} c_{2}\left|\hat{T}^{t_{i}+q_{i}} x-\hat{T}^{t_{i}+q_{i}} y\right|+\log \prod_{j=0}^{l_{i}-1} \frac{\hat{T}^{\prime}\left(\hat{T}^{t_{i}+q_{i}+j} x\right)}{\hat{T}^{\prime}\left(\hat{T}^{t_{i}+q_{i}+j} y\right)} .
\end{aligned}
$$

We estimate the second term by

$$
\begin{aligned}
\log \prod_{j=0}^{l_{i}-1} \frac{\hat{T}^{\prime}\left(\hat{T}^{t_{i}+q_{i}+j} x\right)}{\hat{T}^{\prime}\left(\hat{T}^{t_{i}+q_{i}+j} y\right)} & \leq \sum_{j=0}^{l_{i}-1}|\log | \hat{T}^{\prime}\left(\hat{T}^{t_{i}+q_{i}+j} x\right)||-|\log | \hat{T}^{\prime}\left(\hat{T}^{t_{i}+q_{i}+j} y\right)|| \\
& \leq \sum_{j=0}^{l_{i}-1} \frac{1}{2 a \delta}\left|\hat{T}^{\prime}\left(\hat{T}^{t_{i}+q_{i}+j} x\right)-\hat{T}^{\prime}\left(\hat{T}^{t_{i}+q_{i}+j} y\right)\right| \\
& \leq \sum_{j=0}^{l_{i}-1} \frac{1}{\delta}\left|\hat{T}^{t_{i}+q_{i}+j}(x)-\hat{T}^{t_{i}+q_{i}+j}(y)\right| .
\end{aligned}
$$

We substitute this result back into equation (26) and use Lemma 2.5(a) to get

$$
\begin{aligned}
\log \frac{\left(\hat{T}^{n}\right)^{\prime}(x)}{\left(\hat{T}^{n}\right)^{\prime}(y)} & \leq \sum_{i=0}^{s}\left(\frac{c_{2}}{c_{0}^{\prime} \delta} e^{-\frac{1}{3} \lambda_{0} l_{i}}+\sum_{j=0}^{l_{i}-1} \frac{1}{\delta} \frac{1}{c_{0}^{\prime} \delta} e^{-\frac{1}{3} \lambda_{0}\left(l_{i}-j\right)}\right)\left|\hat{T}^{t_{i+1}}(x)-\hat{T}^{t_{i+1}}(y)\right| \\
& \leq \sum_{i=0}^{s}\left(c_{2} \frac{1}{c_{0}^{\prime} \delta}+\frac{1}{\delta} \frac{1}{c_{0}^{\prime} \delta} \sum_{j=1}^{\infty} e^{-\frac{1}{3} \lambda_{0} j}\right)\left|\hat{T}^{t_{i+1}}(x)-\hat{T}^{t_{i+1}}(y)\right| \\
& =: c_{3} \sum_{i=0}^{s}\left|\hat{T}^{t_{i+1}}(x)-\hat{T}^{t_{i+1}}(y)\right| .
\end{aligned}
$$

We estimate $\left|\hat{T}^{t_{i}}(x)-\hat{T}^{t_{i}}(y)\right|$ using equation (5). Since $\left|\left(\hat{T}^{t_{i+1}-t_{i}}\right)^{\prime}\left(\hat{T}^{t_{i}} x\right)\right| \geq e^{\frac{1}{3} \lambda_{0}\left(t_{i+1}-t_{i}\right)}$, we have

$$
\left|\hat{T}^{t_{i}}(x)-\hat{T}^{t_{i}}(y)\right| \leq e^{-\frac{1}{3} \lambda_{0}\left(t_{i+1}-t_{i}\right)}\left|\hat{T}^{t_{i+1}}(x)-\hat{T}^{t_{i+1}}(y)\right| \leq e^{-\frac{1}{3} \lambda_{0}\left(n-t_{i}\right)}\left|\hat{T}^{n}(x)-\hat{T}^{n}(y)\right| .
$$

We substitute this back into equation (27) to conclude the proof:

$$
\begin{aligned}
\log \frac{\left(\hat{T}^{n}\right)^{\prime}(x)}{\left(\hat{T}^{n}\right)^{\prime}(y)} & \leq c_{3} \sum_{i=0}^{s} e^{-\frac{1}{3} \lambda_{0}\left(n-t_{i+1}\right)}\left|\hat{T}^{n}(x)-\hat{T}^{n}(y)\right| \\
& \leq c_{3}\left|\hat{T}^{n}(x)-\hat{T}^{n}(y)\right| \sum_{i=0}^{\infty} e^{-\frac{1}{6} \lambda_{0} k_{0} i} \\
& =: \tilde{C}\left|\hat{T}^{n}(x)-\hat{T}^{n}(y)\right|
\end{aligned}
$$

Note that the constant $\tilde{C} \sim 1 / \delta^{2}$. Also, this Lemma proves item (c) of Proposition 4.1. 
Item (d) of Proposition 4.1 follows immediately using the assumption that $\varepsilon \leq 1 / 4^{9} \tilde{C}$ and noting that $\left|\hat{T}^{S} x-\hat{T}^{S} y\right|<4^{9} \varepsilon$ since $\left|T^{S-1} \omega\right| \leq 4^{8} \varepsilon$ and $\left|\hat{T}^{\prime}\right| \leq 4$. Since $\left|T^{S} \omega\right| /|\omega| \geq$ $4^{8} / 3$, for any $x \in \omega$ we must have

$$
\left|\left(T^{s}\right)^{\prime}(x)\right| \geq \frac{4^{8}}{3} e^{-\tilde{C} 4^{9} \varepsilon}
$$

from which $\left|\left(T^{S}\right)^{\prime}(x)\right|>4^{6}$ follows.

Remark. The weaker bound

$$
\left|\frac{\left(\hat{T}^{n}\right)^{\prime}(x)}{\left(\hat{T}^{n}\right)^{\prime}(y)}-1\right| \leq \tilde{C}
$$

can be proved using Lemma 4.5(b) instead of equation (25) in equation (26). Although the bound is weaker than that in Lemma 4.6, it is valid for any time $n$ when $\hat{T}^{n} \omega$ is free, not just when $n=S(\omega)$. We shall use this bound later in $\S 5.4$.

\section{An a.c.c.i.m. for the logistic map}

5.1. Defining the tower map. We identify $\Lambda^{(1)}, \ldots, \Lambda^{(N)}$ with $N$ intervals of unit length, $\hat{\Delta}_{0}^{(1)}, \ldots, \hat{\Delta}_{0}^{(N)}$. The partition $\eta$ and return time function $R$ of Proposition 4.2 induce a partition and return time function on $\hat{\Delta}_{0}:=\bigcup_{i} \hat{\Delta}_{0}^{(i)}$ which we refer to by the same names. We define the tower as usual:

$$
\hat{\Delta}:=\left\{(x, n) \in \hat{\Delta}_{0} \times \mathbb{N}: n<R(x)\right\} .
$$

Recall the notation $\hat{\Delta}_{l}=\left.\hat{\Delta}\right|_{n=l}$ for the $l$ th level of the tower, and let $\hat{\Delta}_{l}^{(i)}$ denote the $l$ th level above $\hat{\Delta}_{0}^{(i)}$. Define the projection $\pi_{0}: \hat{\Delta}_{0} \rightarrow I$ as piecewise linear with $\pi_{0}\left(\hat{\Delta}_{0}^{(i)}\right)=\Lambda^{(i)}$ and $\pi_{0}^{\prime}\left|\hat{\Delta}_{0}^{(i)}=\right| \Lambda^{(i)} \mid$. This makes $\varepsilon \leq \pi_{0}^{\prime} \leq 2 \varepsilon$ on $\hat{\Delta}_{0}$. If $\hat{T}^{R} \omega \subset H$, then we put a hole $\tilde{H}_{R(\omega), j}^{(i)}$ in the $R(\omega)$ level of the tower above $\pi_{0}^{-1} \omega$ in $\hat{\Delta}_{0}^{(i)}$. This defines the hole $\tilde{H}$ in the tower $\hat{\Delta}$. Let $\Delta=\hat{\Delta} \backslash \tilde{H}$ and in general $\Delta_{l}^{(i)}=\hat{\Delta}_{l}^{(i)} \backslash \tilde{H}$.

Let $\hat{F}$ be the tower map, $\hat{F}: \Delta \rightarrow \hat{\Delta}$. Define a projection $\pi: \hat{\Delta} \rightarrow[-1,1]$ such that $\pi \circ \hat{F}=\hat{T} \circ \pi$ on $\Delta$. The elements of the Markov partition $\Delta_{l, j}^{(i)}$ are the maximal intervals on $\hat{\Delta}_{l}^{(i)}$ which project onto the dynamically defined elements of $\Omega_{l}$ in the construction of the return time function $R$ above the reference interval $\Lambda^{(i)}$, with the exception that each $\Delta_{0}^{(i)}$ is taken to be a single element of the partition. Recall that $\Delta_{l, j}^{(i) *}$ are the elements which return to $\Delta_{0}$ at time $l+1$.

For $x \in \Delta$, we have the identity $\pi^{\prime} \circ \hat{F}^{l}(x) \cdot\left(\hat{F}^{l}\right)^{\prime}(x)=\left(\hat{T}^{l}\right)^{\prime} \circ \pi(x) \cdot \pi^{\prime}(x)$. If $R(x)>l$ then $\left(\hat{F}^{l}\right)^{\prime}(x)=1$ so

$$
\pi^{\prime} \circ \hat{F}^{l}(x)=\left(\hat{T}^{l}\right)^{\prime} \circ \pi(x) \cdot \pi^{\prime}(x) .
$$

Now let $z \in \Delta_{0}^{(i)}$ with $R(z)=l+1$. Let $\hat{F}^{l} z=\bar{x}$. Then $\pi^{\prime} \circ \hat{F}^{l+1}(z) \cdot\left(\hat{F}^{l+1}\right)^{\prime}(z)=$ $\left(\hat{T}^{l+1}\right)^{\prime} \circ \pi(z) \cdot \pi^{\prime}(z)$. But $\hat{F}^{l+1}(z) \in \Delta_{0}^{(j)}$ so that $\pi^{\prime} \circ \hat{F}^{l+1}(z)=\left|\Lambda^{(j)}\right|$ and $\left(\hat{F}^{l+1}\right)^{\prime}(z)=$ $\hat{F}^{\prime}\left(\hat{F}^{l} z\right) \cdot\left(\hat{F}^{l}\right)^{\prime}(z)=\hat{F}^{\prime}(\bar{x})$. This yields

$$
\hat{F}^{\prime}(\bar{x})=\left(T^{l+1}\right)^{\prime}(\pi z) \frac{\left|\Lambda^{(i)}\right|}{\left|\Lambda^{(j)}\right|} .
$$


Using this fact, Proposition 4.2(d) and equation (6), we conclude that

$$
\inf _{\Delta^{*}}\left|\hat{F}^{\prime}\right|>\frac{4^{6}}{2} \quad \text { and } \quad \inf _{\Delta_{l}^{*}}\left|\hat{F}^{\prime}\right|>\frac{c_{0} c_{0}^{\prime} \delta}{2} e^{\frac{1}{3} \lambda_{0}(l+1)} .
$$

We derive a distortion estimate for $\hat{F}$. Let $x, y \in \Delta_{l, j}^{(k)}$ be such that $\hat{F} x, \hat{F} y \in \hat{\Delta}_{0}^{(i)}$. Using Lemma 4.6 and equation (30) we have

$$
\begin{aligned}
\left|\frac{\hat{F}^{\prime}(x)}{\hat{F}^{\prime}(y)}-1\right| & =\left|\frac{\left(\hat{T}^{l+1}\right)^{\prime}\left(\pi \circ \hat{F}^{-l} x\right)}{\left(\hat{T}^{l+1}\right)^{\prime}\left(\pi \circ \hat{F}^{-l} y\right)}-1\right| \\
& \leq \tilde{C}\left|\hat{T}^{l+1}\left(\pi \circ \hat{F}^{-l} x\right)-\hat{T}^{l+1}\left(\pi \circ \hat{F}^{-l} y\right)\right| \\
& =\tilde{C}|\pi \circ \hat{F}(x)-\pi \circ \hat{F}(y)| \\
& \leq \tilde{C}(2 \varepsilon)|\hat{F}(x)-\hat{F}(y)| .
\end{aligned}
$$

Let $F=\hat{F} \mid\left(\Delta \backslash \hat{F}^{-1} \tilde{H}\right) . F$ also satisfies the relation $\pi \circ F=T \circ \pi$ on its domain, and so the above estimates hold for $F^{\prime}$.

5.2. ( $(\Delta, F, m)$ satisfies Conditions $H 1$ and H2. Recall Properties P2(a)-(c) required of the tower map in $\S 3$ as well as Conditions H1 and H2. It is clear from the discussion of the previous section that $\hat{F}$ has properties (a) and (c) with $C=2 \tilde{C} \varepsilon \leq 2 / 4^{9}$. Property (b) follows from equation (31). Let $v$ be the smallest integer such that $\left(c_{0} c_{0}^{\prime} \delta / 2\right) e^{\frac{1}{6} \lambda_{0}(v+1)} \geq 2$. For $l \geq v,\left|F^{\prime}\right| \geq 2 e^{\frac{1}{6} \lambda_{0}(l+1)}$, while for $l<v,\left|F^{\prime}\right|>2\left(4^{5}\right)^{l / v}$. So (b) follows with $\gamma=2$ and $\beta=\min \left\{\lambda_{0} / 6,(5 / \nu) \log 4\right\}$.

Condition H1 is satisfied with the same $\theta$ as in the statement of Proposition 4.2. This is because $m \hat{\Delta}_{n}=m \Delta_{n}+m \tilde{H}_{n}$ and Proposition 4.2 yields $m \Delta_{n} \leq\left(N C^{\prime \prime} / \varepsilon\right) \theta^{n}$ while Proposition 4.3 yields $m \tilde{H}_{n} \leq(N D m H / \varepsilon) \theta^{n}$.

In $\S 3, \xi$ is defined so that $e^{-\xi}>\max \left\{\theta, e^{-\beta}\right\}$. Actually, from the proof of Proposition 4.2, the rate of contraction of $\theta$ is slower than $e^{-\beta}$ so we may choose $\xi=-\frac{1}{2} \log \theta$. Then Condition $\mathrm{H} 2$ becomes

$$
\sum_{l=1}^{\infty} \theta^{-(l-1) / 2} m \tilde{H}_{l}<\frac{(1-\sqrt{\theta})^{2}}{1+C} .
$$

But $m \tilde{H}_{l} \leq(N D m H / \varepsilon) \theta^{l}$. So

$$
\sum_{l=1}^{\infty} \theta^{-(l-1) / 2} m \tilde{H}_{l} \leq \sum_{l=1}^{\infty} \frac{N D m H}{\varepsilon} \theta^{-(l-1) / 2} \theta^{l} \leq \frac{N D m H}{\varepsilon} \frac{\theta}{1-\sqrt{\theta}} .
$$

Using this estimate, we see that Condition $\mathrm{H} 2$ will be satisfied if $H$ satisfies

$$
m H<\frac{(1-\sqrt{\theta})^{2}}{1+C} \cdot \frac{\varepsilon(1-\sqrt{\theta})}{N D \theta}
$$

which is slightly weaker than Condition A3. 
5.3. Existence and lower bound for an a.c.c.i.m. Since $(\Delta, F, m)$ satisfies Condition $\mathrm{H} 1$ and Condition $\mathrm{H} 2$, we conclude that there exists $\varphi \in X_{M}$ such that $d \tilde{\mu}:=\varphi d m$ is an a.c.c.i.m. with respect to $F$ acting on $\Delta$. Let $\lambda$ be the eigenvalue of $\tilde{\mu}$ and note that $\lambda \geq \sqrt{\theta}$ by the remark following Theorem 3.1. By that same remark we have

$$
\lambda \geq 1-M \sum_{l \geq 1} e^{\xi(l-1)} m\left(\tilde{H}_{l}\right) \geq 1-\frac{N D \theta}{\varepsilon(1-\sqrt{\theta})} m(H)
$$

by the estimates of $\$ 5.2$ based on Condition A3.

Now define a measure $\mu$ on $I$ by

$$
\mu(A):=\tilde{\mu}\left(\pi^{-1} A\right)
$$

for any Borel subset $A$ of $I$. Then $\mu$ will be an a.c.c.i.m. with respect to $T$ with the same eigenvalue $\lambda$ since for any Borel $A \subset I$,

$$
\begin{aligned}
\mu\left(T^{-1} A\right) & :=\tilde{\mu}\left(\pi^{-1} \circ T^{-1} A\right)=\tilde{\mu}\left(F^{-1} \circ \pi^{-1} A\right) \\
& =\lambda \tilde{\mu}\left(\pi^{-1} A\right)=: \lambda \mu(A) .
\end{aligned}
$$

Let $\psi$ be the density of the measure $\mu$. The fact that $\psi$ is bounded away from zero relies on the genericity Condition A4 as well as the following lemma which is proved in a more general case in [Y1] as Lemma 2.1.

Lemma 5.1. Let $\hat{T} \in \mathcal{M}$. For every interval $J \subseteq[-1,1]$, there exists $n=n(J)$ such that $\hat{T}^{n} J \supseteq\left[\hat{T}^{2} 0, \hat{T} 0\right]=[1-a, 1]$.

The integer $n$ in the above lemma can be chosen to depend only on the length of the interval $J$. If we consider only those intervals with length at least $\varepsilon_{0} / 2$, then we can choose a single $n_{0}=n_{0}\left(\varepsilon_{0}\right)$ such that any such interval $J$ satisfies $\hat{T}^{n_{0}} J \supseteq[1-a, 1]$. This is the $n_{0}$ introduced in $\S 2.2$.

For convenience, we recall Condition A4 of $\$ 2.2$.

Condition A4.

(a) $\hat{T}^{i} H_{j} \cap G_{k}=\emptyset$ for all $j, k \in[1, \ldots, L], 0 \leq i \leq n_{0}$.

(b) $\hat{T}^{i} H_{j} \cap H_{k}=\emptyset$ for all $j, k \in[1, \ldots, L], 1 \leq i \leq n_{0}$.

Recall that the intervals $G_{j}$ in the statement of Condition A4 are the symmetric counterparts of the $H_{j}$ so that $\hat{T}^{-1}\left(\hat{T} H_{j}\right)=H_{j} \cup G_{j}$.

LEMMA 5.2. Given any $J \subseteq I$ such that $|J| \geq \varepsilon_{0} / 2$, then

$$
\bigcup_{i=0}^{2 n_{0}} T^{i} J \supseteq[1-a, 1] \backslash H .
$$

Proof. Fix $J$ as in the statement of the lemma. Suppose there exists an interval $\omega$ such that $\omega \cap\left(\bigcup_{i=0}^{n_{0}} T^{i} J\right)=\emptyset$. Since $\omega \subseteq \hat{T}^{n_{0}} J$, we must have $\omega \cap \hat{T}^{i_{k}} H_{k} \neq \emptyset$ for some $H_{k}$

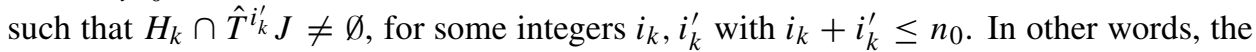
piece of $J$ that should have covered part of $\omega$ fell into $H_{k}$ before time $n_{0}$. In particular, Condition A4(a) implies that $G_{1}, \ldots, G_{L}$ are covered by time $n_{0}$, i.e. $G_{k} \subset T^{n_{0}} J$. 
Condition A4(b) says that $G_{k}$ cannot fall into the hole again before time $n_{0}$ so that $T^{i_{k}} G_{k}=\hat{T}^{i_{k}} H_{k}$. We conclude that the part of $\omega$ which should have been covered by the piece of $J$ that fell into $H_{k}$ is at the latest covered by an iterate of $G_{k}$ at time $n_{0}+i_{k}$. Doing this for each $k$, we have $\omega \subset \bigcup_{k=1}^{L} T^{i_{k}} G_{k}$ and so $\omega \subset \bigcup_{i=0}^{2 n_{0}} T^{i} J$.

In $\S 4.1$, we showed that every interval of length at least $\varepsilon$ grows to length $4^{8} \varepsilon$ in exponential time that depends only on $\varepsilon$. In fact, the construction holds as long as the interval remains less than length $\varepsilon_{0}$ due to Condition A2. This allows us to conclude that every interval of length $\varepsilon$ grows to length $\varepsilon_{0}$ in exponential time and from there, by Lemma 5.2, it covers $[1-a, 1]$ by time $2 n_{0}$. This implies that the density $\psi$ is bounded away from zero on $[1-a, 1] \backslash H$.

5.4. Shape of the density and proof of Theorem 2.4. In this section, we derive bounds on the density $\psi$ and show that it has the form given in the statement of Theorem 2.3. Since the bounds depend only on $H=H_{t}$ in a sequence of holes of the form described in Theorem 2.4, they are uniform in $s$ and allow us to prove Theorem 2.4.

Let $\hat{T}$ and $\left\{H_{s}\right\}$ be as in the statement of Theorem 2.4. Let $I_{s}=\hat{I} \backslash H_{s}$ and $T_{s}=$ $\hat{T} \mid I_{s} \cap \hat{T}^{-1} I_{s}$. The assumptions on the holes imply that each $H_{s}$ satisfies Conditions A1-A4 with the same choice of constants. This is because the intervals of monotonicity of the map $T_{s}$ only increase in length as $s \rightarrow 0$. So we may apply Theorems 2.2 and 2.3 for each $s$.

Let $\varphi_{s}$ be the conditionally invariant density for $\tilde{\mu}_{s}$ on $\Delta$ with eigenvalue $\lambda_{s}$. Let $\psi_{s}$ be the density for $\mu_{s}$, the conditionally invariant measure for $T_{s}$ on $I_{s}$. We fix $s$ and show that $\psi_{s}$ has lower and upper bounds that are independent of $s$.

Lower Bound. Recall that $\varphi_{s} \in X_{M}$ where $M=(1+C) /(1-\sqrt{\theta})$. Thus

$$
\begin{aligned}
1 & =\sum_{l=0}^{\infty} \int_{\Delta_{l}} \varphi_{s} d m \leq \sum_{l=0}^{\infty} \sup _{\Delta_{l}} \varphi_{s} m \Delta_{l} \leq \sup _{\Delta_{0}} \varphi_{s} \sum_{l=0}^{\infty} \frac{1}{\lambda_{s}^{l}} m \Delta_{l} \\
& \leq \sup _{\Delta_{0}} \varphi_{s} \sum_{l=0}^{\infty} \frac{C^{\prime \prime} N}{\varepsilon \lambda_{s}^{l}} \theta^{l} \leq \frac{C^{\prime \prime}}{\varepsilon^{2}} \frac{1}{1-\sqrt{\theta}} \sup _{\Delta_{0}} \varphi_{s}
\end{aligned}
$$

since $\lambda_{s} \geq \sqrt{\theta}$. This implies that there exists an $i$ such that

$$
\sup _{\Delta_{0}^{(i)}} \varphi_{s} \geq \frac{\varepsilon^{2}(1-\sqrt{\theta})}{C^{\prime \prime}} .
$$

The regularity of $\varphi_{s}$ yields a lower bound on the density,

$$
\inf _{\Delta_{0}^{(i)}} \varphi_{s} \geq \frac{\varepsilon^{2}(1-\sqrt{\theta})}{C^{\prime \prime}(1+M)}
$$

which in turn implies

$$
\inf _{\Lambda^{(i)}} \psi_{s} \geq \frac{\varepsilon(1-\sqrt{\theta})}{2 C^{\prime \prime}(1+M)} .
$$


Since the length scale $\varepsilon_{0}$ can be chosen independent of $s$ (if $\varepsilon_{0}$ works for $H_{t}$, it will automatically work for each $H_{s}$ in the sequence), the constant $n_{0}$ of Lemma 5.2 is independent of $s$. The length scale $\varepsilon$ is also independent of $s$ so we conclude that $\Lambda^{(i)}$ will grow to cover $[1-a, 1] \backslash H_{S}$ in a fixed number of iterates depending only on $\varepsilon$ and $\varepsilon_{0}$. Call this number $N_{0}$.

For any $x \in[1-a, 1] \backslash H_{s}$, there exists $z \in \Lambda^{(i)}$ and $n \leq N_{0}$ such that $T_{s}^{n}(z)=x$. Let $\mathcal{P}_{s}$ be the Perron-Frobenius operator associated with the map $T_{s}$. Then

$$
\lambda_{s}^{n} \psi_{s}(x)=\mathcal{P}_{s}^{n} \psi_{s}(x)=\sum_{y \in T_{s}^{-n} x} \frac{\psi_{s}(y)}{\left|\left(T_{s}^{n}\right)^{\prime}(y)\right|} \geq \frac{\psi_{s}(z)}{\left|\left(T_{s}^{n}\right)^{\prime}(z)\right|} .
$$

So

$$
\inf _{[1-a, 1] \backslash H_{s}} \psi_{s} \geq \frac{\varepsilon(1-\sqrt{\theta})}{4^{N_{0}} 2(1+M) C^{\prime \prime}},
$$

which is a lower bound independent of $s$.

Upper Bound. For the upper bound, we first estimate the number of preimages under the projection $\pi$ a point in $I$ can have on any given level of the tower $\Delta$. To do this, we consider how many unreturned pieces can be generated while iterating one of the reference intervals $\Lambda^{(i)}$. Once a piece is returned, it no longer generates preimages on subsequent levels of the tower. There are several ways that pieces can be generated.

(1) An interval intersects the hole and is cut into two pieces. This can happen at most once every $m_{0}$ iterates by Condition A2.

(2) An interval grows to length $4^{8} \epsilon$ and the stopping time $S$ is declared. Most of this interval is returned, except for the two end pieces which continue to be iterated. Thus up to two new pieces are formed. Since each piece begins with length less than $3 \varepsilon$ and must grow to length $4^{8} \varepsilon$ before another stopping time is declared, this can only happen once every eight iterates.

(3) An interval lands on 0 , the critical point. Then we consider that two new pieces are formed, one on each side of 0 . This can happen at most once every $p\left(k_{0}\right)$ iterates. Note that $p\left(k_{0}\right) \geq k_{0} / 2 \geq 25$.

(4) An interval which lands in $(-\delta, \delta)$ reaches its recovery time. Suppose a piece $\omega$ is mapped onto an interval extending from $I_{r}$ to $I_{S}$ at time $t$. We label the subinterval of $\omega$ which lands in $I_{k}$ at time $t$ as $\omega_{k}$. Without loss of generality, assume $0<s \leq r \leq \infty$. We consider $\omega$ as one piece from time $t$ until time $t+q(s)$. At time $t+q(s), \omega_{s}$ is counted as a separate piece. If $\left|T^{t+q(s)} \omega_{s}\right|<4^{8} \varepsilon$, then we simply continue to iterate it. It will generate new pieces at the rate described by items (1)-(3) above until the next time it enters $(-\delta, \delta)$. If $\left|T^{t+q(s)} \omega_{s}\right| \geq 4^{8} \varepsilon$, then by definition of the stopping time $S$ after Proposition 4.1 and the stopping time $R$ described after Proposition 4.2, only one new piece will not be returned at time $t+q(s)$ (as opposed to the usual two pieces which are not returned when $R$ is declared on the middle part of an interval). This is because in the construction, the piece of $\omega_{s}$ which does not completely cover the last $\Lambda^{(j)}$ on the side near $\omega_{s+1}$ is adjoined to $\omega_{s+1}$ and the stopping time $S$ is not declared on this piece until time $t+q(s+1)$. Thus returns of this type generate at most linear growth in the number of pieces which can overlap at any given time. 
We see from these considerations that the number of pieces which can overlap at time $n$ and are generated by the single interval $\Lambda^{(i)}$ satisfies the following bound:

$$
\#\{\text { Pieces }\} \leq n 2^{\left(n / m_{0}+n / 8+n / p\left(k_{0}\right)\right)+3} \leq 8 n 2^{53 n / 200},
$$

where we have used the fact that $m_{0} \geq 10$ and $p\left(k_{0}\right) \geq 25$.

We denote by $\pi_{l, j}^{(i)-1}$ the inverse of $\pi \mid \Delta_{l, j}^{(i)}$. Let $\psi=\psi_{s}$ and $\varphi=\varphi_{s}$ for any $s \in[0, t]$. The density $\psi$ can be written as

$$
\psi(x)=\sum_{i=1}^{N} \sum_{l} \sum_{j} \frac{\varphi\left(\pi_{l, j}^{(i)-1} x\right)}{\pi^{\prime}\left(\pi_{l, j}^{(i)-1} x\right)} .
$$

We seek to estimate this sum by determining the growth of $\pi^{\prime}\left(\pi_{l, j}^{(i)-1} x\right)$. In general we have the relation $\pi^{\prime}\left(F^{l} y\right) \cdot\left(F^{l}\right)^{\prime}(y)=\left(T^{l}\right)^{\prime}(\pi y) \cdot \pi^{\prime}(y)$. Letting $y=F^{-l}\left(\pi_{l, j}^{(i)-1} x\right) \in \Delta_{0}$, we have $\left(F^{l}\right)^{\prime}(y)=1$ and $\pi^{\prime}(y)=\left|\Lambda^{(i)}\right|$ so that

$$
\pi^{\prime}\left(\pi_{l, j}^{(i)-1} x\right)=\left(T^{l}\right)^{\prime}(\pi y) \cdot\left|\Lambda^{(i)}\right| .
$$

Let $l_{j}$ be the smallest positive integer $k \leq l$ such that $\pi \circ F^{-k}\left(\pi_{l, j}^{(i)-1} x\right) \in(-\delta, \delta)$. If no such $k$ exists, then set $l_{j}=l$ and do Case 1 below.

Case 1. If $l_{j} \geq p\left(\pi \circ F^{-l_{j}}\left(\pi_{l, j}^{(i)-1} x\right)\right)$, then $\pi\left(\Delta_{l, j}^{(i)}\right)$ is free so that $\left|\left(T^{l}\right)^{\prime}(\pi y)\right| \geq$ $c_{0} c_{0}^{\prime} \delta e^{\frac{1}{3} \lambda_{0} l}$, using equation (6).

Case 2. If $l_{j}<p\left(\pi \circ F^{-l_{j}}\left(\pi_{l, j}^{(i)-1} x\right)\right)$, then we estimate $\left(T^{l}\right)^{\prime}(\pi y)$ as follows:

$$
\left(T^{l}\right)^{\prime}(\pi y)=\left(\hat{T}^{l_{j}}\right)^{\prime}\left(T^{l-l_{j}}(\pi y)\right) \cdot\left(\hat{T}^{l-l_{j}}\right)^{\prime}(\pi y) .
$$

The second factor in the above expression is greater than or equal to $c_{0} c_{0}^{\prime} \delta e^{\frac{1}{3} \lambda_{0}\left(l-l_{j}\right)}$ since $\hat{T}^{l-l_{j}-1}(\pi y)$ is free. To estimate the first factor, we use equation (23) of $\S 4.5$ to note that for $z \in(-\delta, \delta)$,

$$
\begin{aligned}
\left|\left(\hat{T}^{n}\right)^{\prime}(z)\right| & =\hat{T}^{\prime}(z) \cdot\left(\hat{T}^{n-1}\right)^{\prime}(\hat{T} z) \geq 2 a|z| \cdot \frac{1}{d_{0}}(1.9)^{n-1} \\
& \geq 2 a \sqrt{\frac{\left|\hat{T}^{n} z-\hat{T}^{n} 0\right|}{d_{0}(1.9)^{n-1}}} \cdot \frac{1}{d_{0}}(1.9)^{n-1}=\frac{2 a}{d_{0}^{3 / 2}}(1.9)^{(n-1) / 2} \sqrt{\left|\hat{T}^{n} z-\hat{T}^{n} 0\right|} .
\end{aligned}
$$

Thus

$$
\left(T^{l}\right)^{\prime}(\pi y) \geq d_{0}^{\prime}(1.9)^{\left(l_{j}-1\right) / 2} e^{\frac{1}{3} \lambda_{0}\left(l-l_{j}\right)} \sqrt{\left|x-\hat{T}^{l_{j}} 0\right|},
$$

where $d_{0}^{\prime}=\left(2 a / d_{0}^{3 / 2}\right) c_{0} c_{0}^{\prime} \delta$.

Let $p_{l, j}^{(i)}=p\left(\pi \circ F^{-l_{j}}\left(\pi_{l, j}^{(i)-1} x\right)\right)$. Recall that $\varphi \leq M / \lambda_{s}^{l} \leq M e^{l / 42}$ on $\Delta_{l}$. Putting Cases 1 and 2 together, we have the following bound on $\psi(x)$ :

$$
\psi(x) \leq \sum_{i} \sum_{\Delta_{l, j}^{(i)}: l_{j} \geq p_{l, j}^{(i)}} \frac{M e^{l / 42}}{c_{0} c_{0}^{\prime} \delta \varepsilon} e^{-\frac{1}{3} \lambda_{0} l}+\sum_{i} \sum_{\Delta_{l, j}^{(i)}: l_{j}<p_{l, j}^{(i)}} \frac{2 M e^{\frac{l}{42}} e^{-\frac{1}{3} \lambda_{0}\left(l-l_{j}\right)}(1.9)^{-l_{j} / 2}}{d_{0}^{\prime} \varepsilon \sqrt{\left|x-\hat{T}^{l_{j}} 0\right|}}
$$


Using equation (34), we see that the first sum is less than

$$
\sum_{i} \sum_{l} \frac{M e^{l / 42}}{c_{0} c_{0}^{\prime} \delta \varepsilon} e^{-\frac{1}{3} \lambda_{0} l} 8 l 2^{53 l / 200}<\infty
$$

if we take $\lambda_{0}=\log 1.9$.

We fix $i$ and estimate the second term by

$$
\begin{aligned}
& \sum_{\substack{\Delta_{l, j}^{(i)} \\
l_{j}<p_{l, j}^{(i)}}} \frac{2 M e^{l / 42} e^{-\frac{1}{3} \lambda_{0}\left(l-l_{j}\right)}(1.9)^{-l_{j} / 2}}{d_{0}^{\prime} \varepsilon \sqrt{\left|x-\hat{T}^{l_{j}} 0\right|}} \\
& \quad \leq \sum_{k} \sum_{\Delta_{l, j}^{(i)}: l_{j}=k} \frac{2 M e^{l / 42} e^{-\frac{1}{3} \lambda_{0}(l-k)}(1.9)^{-k / 2}}{d_{0}^{\prime} \varepsilon \sqrt{\left|x-\hat{T}^{k} 0\right|}} \\
& \leq \sum_{k, l} \frac{2 M e^{l / 42} e^{-\frac{1}{3} \lambda_{0}(l-k)}(1.9)^{-k / 2}}{d_{0}^{\prime} \varepsilon \sqrt{\left|x-\hat{T}^{k} 0\right|}} 8(l-k) 2^{53(l-k) / 200},
\end{aligned}
$$

where in the second line we have used the fact that the number of pieces we are summing over from time $k$ to time $l$ has not changed since these pieces are bound during that time. Using $\lambda_{0}=\log 1.9$, we have $e^{-\frac{1}{3} \lambda_{0}(l-k)} 2^{53(l-k) / 200} \leq e^{-(l-k) / 34}$. So the sum becomes

$$
\sum_{k} \sum_{l} \frac{16 M l e^{l / 42} e^{-(l-k) / 34}(1.9)^{-k / 2}}{d_{0}^{\prime} \varepsilon \sqrt{\left|x-\hat{T}^{k} 0\right|}}=\sum_{k} \frac{16 M e^{k / 34}(1.9)^{-k / 2}}{d_{0}^{\prime} \varepsilon \sqrt{\left|x-\hat{T}^{k} 0\right|}} \sum_{l} l e^{-l / 34} e^{l / 42}
$$

and both series converge.

$\rho_{1}$ has bounded variation. Note that $p_{l, j}^{(i)}$ is constant on $\Delta_{l, j}^{(i)}$. We set

$$
\rho_{1}=\sum_{\Delta_{l, j}^{(i)}: l_{j} \geq p_{l, j}^{(i)}} \frac{\varphi \circ \pi_{l, j}^{(i)-1}}{\pi^{\prime} \circ \pi_{l, j}^{(i)-1}} .
$$

Equation (35) and equation (28) of $\S 4.5$ imply that

$$
\left|\frac{\left(\pi_{l, j}^{(i)-1}\right)^{\prime}(x)}{\left(\pi_{l, j}^{(i)-1}\right)^{\prime}(y)}-1\right| \leq \tilde{C}
$$

which in turns yields the bound we need to estimate the variation:

$$
\left|\frac{\left(\pi_{l, j}^{(i)-1}\right)^{\prime \prime}(x)}{\left(\pi_{l, j}^{(i)-1}\right)^{\prime}(x)}\right| \leq \tilde{C}
$$

Let $\bigvee_{J} f$ denote the variation of a function on the interval $J$. Recall that the regularity functional on the tower is given by $\left\|\left.f\right|_{\Delta_{l, j}^{(i)}}\right\|_{r}=\sup _{\Delta_{l, j}^{(i)}}\left|f^{\prime} / f\right| e^{-\xi l}$ for any $f \in X$. Thus

$$
\bigvee_{\Delta_{l, j}^{(i)}} f=\int_{\Delta_{l, j}^{(i)}}\left|f^{\prime}\right| d m \leq\left\|\left.f\right|_{\Delta_{l, j}^{(i)}}\right\|_{r} e^{\xi l} \int_{\Delta_{l, j}^{(i)}}|f| d m .
$$


We now estimate the variation of $\rho_{1}$ on $I$ :

$$
\bigvee_{I} \rho_{1} \leq \sum_{\Delta_{l, j}^{(i)}: l_{j} \geq p_{l, j}^{(i)} \pi\left(\Delta_{l, j}^{(i)}\right)} \varphi \circ \pi_{l, j}^{(i)-1} \cdot\left(\pi_{l, j}^{(i)-1}\right)^{\prime}+2\left|\frac{\varphi \circ \pi_{l, j}^{(i)-1}}{\pi^{\prime} \circ \pi_{l, j}^{(i)-1}}\right|_{\infty}
$$

Since the $\Delta_{l, j}^{(i)}$ in the sum are free, we estimate the second term using

$$
\left|\frac{\varphi \circ \pi_{l, j}^{(i)-1}}{\pi^{\prime} \circ \pi_{l, j}^{(i)-1}}\right|_{\infty} \leq \frac{M e^{-\frac{1}{3} \lambda_{0} l}}{\lambda^{l} c_{0}^{\prime} c_{0} \delta}
$$

We estimate the first term one $\Delta_{l, j}^{(i)}$ at a time:

$$
\begin{aligned}
\bigvee_{\pi\left(\Delta_{l, j}^{(i)}\right)} \varphi \circ \pi_{l, j}^{(i)-1} \cdot\left(\pi_{l, j}^{(i)-1}\right)^{\prime}= & \int_{\pi\left(\Delta_{l, j}^{(i)}\right)}\left|\left(\varphi \circ \pi_{l, j}^{(i)-1} \cdot\left(\pi_{l, j}^{(i)-1}\right)^{\prime}\right)^{\prime}\right| d m \\
\leq & \int_{\pi\left(\Delta_{l, j}^{(i)}\right)}\left|\varphi^{\prime} \circ \pi_{l, j}^{(i)-1} \cdot\left(\left(\pi_{l, j}^{(i)-1}\right)^{\prime}\right)^{2}\right| d m \\
& +\int_{\pi\left(\Delta_{l, j}^{(i)}\right)}\left|\varphi \circ \pi_{l, j}^{(i)-1} \cdot\left(\pi_{l, j}^{(i)-1}\right)^{\prime \prime}\right| d m \\
\leq & \frac{e^{-\frac{1}{3} \lambda_{0} l}}{c_{0}^{\prime} c_{0} \delta} \int_{\Delta_{l, j}^{(i)}}\left|\varphi^{\prime}\right| d m+\tilde{C} \int_{\Delta_{l, j}^{(i)}} \varphi d m
\end{aligned}
$$

where we have used equation (37) for the second term in the last line. We use equation (38) for the first term in the last line to obtain

$$
\bigvee_{\pi\left(\Delta_{l, j}^{(i)}\right)} \varphi \circ \pi_{l, j}^{(i)-1} \cdot\left(\pi_{l, j}^{(i)-1}\right)^{\prime} \leq \frac{M e^{\left(-\frac{1}{3} \lambda_{0}+\xi\right) l}}{c_{0}^{\prime} c_{0} \delta} \int_{\Delta_{l, j}^{(i)}} \varphi d m+\tilde{C} \int_{\Delta_{l, j}^{(i)}} \varphi d m
$$

Now putting together equations (39) and (40) we conclude that

$$
\bigvee_{I} \rho_{1} \leq \sum_{\Delta_{l, j}^{(i)}: l_{j} \geq p_{l, j}^{(i)}} \frac{M e^{\left(-\frac{1}{3} \lambda_{0}+\xi\right) l}}{c_{0}^{\prime} c_{0} \delta} \int_{\Delta_{l, j}^{(i)}} \varphi d m+\tilde{C} \int_{\Delta_{l, j}^{(i)}} \varphi d m+2 \frac{M e^{-\frac{1}{3} \lambda_{0} l}}{c_{0}^{\prime} c_{0} \delta \lambda^{l}}
$$

This sum is finite since on each level of the tower, there are only finitely many $\Delta_{l, j}^{(i)}$ which are free and it is only these $\Delta_{l, j}^{(i)}$ which we are summing over. The number of such pieces on a level $l$ of the tower has been shown in equation (34) to be bounded by $8 l 2^{53 l / 200}$. Using this estimate, the fact that $\lambda \geq e^{-\frac{1}{42}}$ and the observation that $\xi$ is much smaller than $\lambda_{0}$, the above series is finite and so $\rho_{1}$ has bounded variation.

These estimates show that the density $\psi$ has the form claimed in the statement of Theorem 2.3. Moreover, equation (36) allows us to write $\psi_{s} \leq g$, where $g \in L^{1}(\hat{I})$ and $g$ is independent of $s$ in the sequence of holes.

Since the upper and lower bounds on $\psi_{s}$ are uniform in $s$, we may conclude that the sequence $\left\{\mu_{s}\right\}$ has a subsequence $\left\{\mu_{s_{k}}\right\}$ which converges weakly to a measure $v$ that is 
bounded away from zero on $[1-a, 1]$. The limit measure $v$ cannot be singular because of the uniform upper bound we derived for the sequence $\left\{\psi_{s}\right\}$. Also, since by equation (32) $\lambda_{s} \rightarrow 1$ as $s \rightarrow 0$, we have for any Borel subset $A$ of $[-1,1]$,

$$
\begin{aligned}
v\left(\hat{T}^{-1} A\right) & =\lim _{k \rightarrow \infty} \mu_{s_{k}}\left(\hat{T}^{-1} A\right)=\lim _{k \rightarrow \infty} \mu_{s_{k}}\left(T_{s_{k}}^{-1}\left(A \backslash H_{s_{k}}\right)\right) \\
& =\lim _{k \rightarrow \infty} \lambda_{s_{k}} \mu_{s_{k}}\left(A \backslash H_{s_{k}}\right)=v(A)
\end{aligned}
$$

so that $v$ is an absolutely continuous invariant measure for $\hat{T}$ with density bounded away from zero on $[1-a, 1]$. But there is only one such measure for $\hat{T}$ ([Y1]). This implies that the entire sequence $\left\{\mu_{s}\right\}$ converges to the unique ergodic a.c.i.m. for $\hat{T}$ as $m\left(H_{s}\right) \rightarrow 0$.

Acknowledgement. The author is grateful to L.-S. Young for many helpful discussions and guidance during the writing of this paper.

\section{REFERENCES}

[BC] M. Benedicks and L. Carleson. On iterates of $1-a x^{2}$ on $(-1,1)$. Ann. Math. 122 (1985), 1-25.

[BCh] H. van den Bedem and N. Chernov. Expanding maps of an interval with holes. Ergod. Th. \& Dynam. Sys. 22 (2002), 637-654.

[BK] V. Baladi and G. Keller. Zeta functions and transfer operators for piecewise monotonic transformations. Comm. Math. Phys. 127 (1990), 459-477.

[BY] M. Benedicks and L.-S. Young. Markov extensions and decay of correlations for certain Hénon maps. Astérisque 261 (2000), xi, 13-56.

[C1] N. N. Čencova. A natural invariant measure on Smale's horseshoe. Soviet Math. Dokl. 23 (1981), 87-91.

[C2] N. N. Čencova. Statistical properties of smooth Smale horseshoes. Mathematical Problems of Statistical Mechanics and Dynamics. Ed. R. L. Dobrushin. Reidel, Dordrecht, 1986, pp. 199-256.

[Ch1] N. Chernov. Statistical properties of piecewise smooth hyperbolic systems in high dimensions. Discr. Cont. Dynam. Syst. 5 (1999), 425-448.

[Ch2] N. Chernov. Decay of correlations and dispersing billiards. J. Stat. Phys. 94 (1999), 513-556.

[Ch3] N. Chernov. Sinai billiards under small external forces. Ann. Henri Poincaré 2 (2001), 197-236.

[CM1] N. Chernov and R. Markarian. Ergodic properties of Anosov maps with rectangular holes. Bol. Soc. Bras. Mat. 28 (1997), 271-314.

[CM2] N. Chernov and R. Markarian. Anosov maps with rectangular holes. Nonergodic cases. Bol. Soc. Bras. Mat. 28 (1997), 315-342.

[CMS1] P. Collet, S. Martínez and B. Schmitt. The Yorke-Pianigiani measure and the asymptotic law on the limit Cantor set of expanding systems. Nonlinearity 7 (1994), 1437-1443.

[CMS2] P. Collet, S. Martínez and B. Schmitt. Quasi-stationary distribution and Gibbs measure of expanding systems. Instabilities and Nonequilibrium Structures V. Eds. E. Tirapegui and W. Zeller. Kluwer, Dordrecht, 1996, pp. 205-219.

[CMT1] N. Chernov, R. Markarian and S. Troubetskoy. Conditionally invariant measures for Anosov maps with small holes. Ergod. Th. \& Dynam. Sys. 18 (1998), 1049-1073.

[CMT2] N. Chernov, R. Markarian and S. Troubetskoy. Invariant measures for Anosov maps with small holes. Ergod. Th. \& Dynam. Sys. 20 (2000), 1007-1044.

[D] M. Demers. Markov extensions for dynamical systems with holes: an application to expanding maps of the interval. Israel J. Math. 146 (2005), 189-221.

[KL] G. Keller and C. Liverani. Stability of the spectrum for transfer operators. Ann. Scuola Norm. Sup. Pisa Cl. Sci. (4) 28 (1999), 141-152.

[LiM] C. Liverani and V. Maume-Deschamps. Lasota-Yorke maps with holes: conditionally invariant probability measures and invariant probability measures on the survivor set. Ann. Henri Poincaré Prob. Stat. 39 (2003), 385-412. 
[LM] A. Lopes and R. Markarian. Open billiards: Cantor sets, invariant and conditionally invariant probabilities. SIAM J. Appl. Math. 56 (1996), 651-680.

[PY] G. Pianigiani and J. Yorke. Expanding maps on sets which are almost invariant: decay and chaos. Trans. Amer. Math. Soc. 252 (1979), 351-366.

[R] P. A. Richardson, Jr. Natural measures on the unstable and invariant manifolds of open billiard dynamical systems. Doctoral Dissertation, Department of Mathematics, University of North Texas, 1999.

[WY1] Q. Wang and L.-S. Young. Strange attractors with one direction of instability. Comm. Math. Phys. 218 (2001), 1-97.

[WY2] Q. Wang and L.-S. Young. Nonuniformly expanding one-dimensional maps. Preprint, 2005.

[Y1] L.-S. Young. Decay of correlations for certain quadratic maps. Comm. Math. Phys. 146 (1992), 123-138.

[Y2] L.-S. Young. Statistical properties of dynamical systems with some hyperbolicity. Ann. Math. 147 (1998), 585-650.

[Y3] L.-S. Young. Recurrence times and rates of mixing. Israel J. Math. 110 (1999), 153-188. 